\title{
Sur une conjecture de Mukai
}

\author{
Laurent Bonavero, Cinzia Casagrande, Olivier Debarre et Stéphane Druel
}

Résumé. Généralisant une question de Mukai, nous conjecturons qu'une variété de Fano $X$ de nombre de Picard $\rho_{X}$ et de pseudo-indice $\iota_{X}$ vérifie $\rho_{X}\left(\iota_{X}-1\right) \leq \operatorname{dim}(X)$. Nous démontrons cette conjecture dans plusieurs situations : $X$ est une variété de Fano de dimension $\leq 4, X$ est une variété de Fano torique de dimension $\leq 7$ ou $X$ est une variété de Fano torique de dimension arbitraire avec $\iota_{X} \geq \operatorname{dim}(X) / 3+1$. Enfin, nous présentons une approche nouvelle pour le cas général.

Abstract. Generalizing a question of Mukai, we conjecture that a Fano manifold $X$ with Picard number $\rho_{X}$ and pseudo-index $\iota_{X}$ satisfies $\rho_{X}\left(\iota_{X}-1\right) \leq \operatorname{dim}(X)$. We prove this inequality in several situations : $X$ is a Fano manifold of dimension $\leq 4, X$ is a toric Fano manifold of dimension $\leq 7$ or $X$ is a toric Fano manifold of arbitrary dimension with $\iota_{X} \geq \operatorname{dim}(X) / 3+1$. Finally, we offer a new approach to the general case.

Mathematics Subject Classification (2000). 14J45, 14E30, 14M25.

Mots clés. Variétés de Fano, théorie de Mori, géométrie torique.

\section{Introduction}

Soit $X$ une variété de Fano, c'est-à-dire une variété projective lisse dont le fibré anticanonique $-K_{X}$ est ample, définie sur $\mathbb{C}$ (certains des résultats ci-dessous sont valables en toute caractéristique). Notons $\iota_{X}$ le pseudo-indice de $X$, c'est-à-dire le plus petit entier de la forme $-K_{X} \cdot C$ où $C$ est une courbe rationnelle de $X$, et $\rho_{X}$ le nombre de Picard de $X$. L'indice $r_{X}$ de $X$ est le plus grand entier $m$ tel qu'il existe un fibré en droites $L$ satisfaisant $-K_{X}=m L$ dans $\operatorname{Pic}(X)$; c'est un nombre particulièrement adapté à l'étude des variétés de Fano $X$ avec $\rho_{X}=1$, tandis le pseudo-indice nous semble plus adapté à l'étude des variétés de Fano $X$ avec $\rho_{X} \geq 2$ (penser par exemple aux produits d'espaces projectifs). Mukai a proposé dans [Mu88] l'inégalité $\rho_{X}\left(r_{X}-1\right) \leq \operatorname{dim}(X)$, vérifiée en dimension $\leq 3$ à l'aide de la classification. Nous proposons plus généralement la conjecture suivante.

Conjecture. Si X est une variété de Fano, on a l'inégalité

$$
\rho_{X}\left(\iota_{X}-1\right) \leq \operatorname{dim}(X)
$$


avec égalité si et seulement si $X \simeq\left(\mathbb{P}^{\iota_{X}-1}\right)^{\rho_{X}}$.

Cette conjecture est en partie motivée par le théorème suivant.

Théorème ([Wi90]). Soit $X$ une variété de Fano de dimension $n$, d'indice $r_{X}$ et de pseudo-indice $\iota_{X}$. Si $2 \iota_{X}>n+2$, on a $\rho_{X}=1$. Si $2 r_{X}=n+2$, on a $\rho_{X}=1$ sauf si $X \simeq\left(\mathbb{P}^{\iota} X-1\right)^{2}$.

D'après la théorie de Mori, une variété de Fano $X$ de dimension $n$ vérifie toujours $\iota_{X} \leq n+1$. Si $\iota_{X}=n+1$, on a $X \simeq \mathbb{P}^{n}$ d'après un résultat de Cho, Miyaoka et Shepherd-Barron ([CMS00]) dont une démonstration simplifiée se trouve dans [Ke01].

Dans ce travail, on se propose de montrer l'inégalité $(*)$ lorsque $X$ est une variété de Fano de dimension 3 ou 4 (l'inégalité $(*)$ est vérifiée directement en dimension 2) et lorsque $X$ est une variété de Fano torique de pseudo-indice au moins égal à $\operatorname{dim}(X) / 3+1$.

L'inégalité $(*)$ peut être vérifiée directement pour les variétés de Fano de dimension 4 et d'indice au moins 2, dont la classification est établie par exemple dans [IP99].

Nous démontrons, sans utiliser les résultats de classification des variétés de Fano de dimension 3 ou 4, le résultat suivant, nouveau dans le cas des variétés d'indice 1 et de pseudo-indice au moins 2 .

Théorème. Si $X$ est une variété de Fano de dimension 3 ou 4 , de pseudo-indice $\iota_{X}$ et de nombre de Picard $\rho_{X}$, on a $\rho_{X}\left(\iota_{X}-1\right) \leq \operatorname{dim}(X)$. Si $\rho_{X}\left(\iota_{X}-1\right)=\operatorname{dim}(X)$, on $a X \simeq\left(\mathbb{P}^{\iota_{X}-1}\right)^{\rho_{X}}$.

Dans la situation torique, nos résultats principaux sont les suivants.

Théorème. Soit $X$ une variété de Fano torique de dimension n, de pseudo-indice $\iota_{X}$ et de nombre de Picard $\rho_{X}$. Si $3 \iota_{X} \geq n+3$, on a $\rho_{X}\left(\iota_{X}-1\right) \leq n$. Si de plus $\rho_{X}\left(\iota_{X}-1\right)=n$, on $a \simeq \simeq\left(\mathbb{P}^{\iota_{X}-1}\right)^{\rho_{X}}$.

Théorème. Soit $X$ une variété de Fano torique de dimension $n \leq 7$, de pseudoindice $\iota_{X}$ et de nombre de Picard $\rho_{X}$. On a $\rho_{X}\left(\iota_{X}-1\right) \leq n$. Si $\rho_{X}\left(\iota_{X}-1\right)=n$, on $a X \simeq\left(\mathbb{P}^{\iota_{X}-1}\right)^{\rho_{X}}$.

Les méthodes utilisées sont celles de la théorie de Mori, dont nous rappelons plus bas quelques uns des résultats ou définitions. Mentionnons les trois résultats suivants obtenus au cours de la démonstration des théorèmes précédents et qui ont leur intérêt propre.

Proposition. Soit $X$ une variété de Fano de dimension n, de nombre de Picard 
$\rho_{X} \geq n$ et de pseudo-indice $\iota_{X} \geq 2$. Si toutes les contractions extrémales de $X$ sont des fibrations, $X$ est isomorphe à $\left(\mathbb{P}^{1}\right)^{n}$.

Théorème. Soit $X$ une variété de Fano de dimension $n \geq 4$. Si toutes les contractions extrémales de $X$ sont ou bien des éclatements lisses de centre une courbe lisse ou bien des fibrations de dimension relative 1 et que $X$ possède au moins une contraction extrémale birationnelle, on a $\rho_{X} \leq 3$.

Théorème. Soient $X$ une variété de Fano de dimension $n$ et $Z$ une variété lisse de dimension $m$ avec $\iota_{X} \geq m+1$. S'il existe un morphisme surjectif à fibres connexes $f: X \rightarrow Z$, alors $Z$ est isomorphe à $\mathbb{P}^{m}$ et il existe une variété lisse $Y$ de dimension $n-m$ telle que $X$ est isomorphe à $Y \times \mathbb{P}^{m}$.

Nous présentons enfin une approche possible, en toute dimension, où l'inégalité (*) découlerait de l'existence de certaines chaînes de courbes rationnelles. Le résultat suivant fournit une motivation supplémentaire en direction de la conjecture de Mukai généralisée.

Théorème. Soit $X$ une variété de Fano de dimension n, de nombre de Picard $\rho_{X}$ et de pseudo-indice $\iota_{X}$. S'il existe des familles propres irréductibles $V^{1}, \ldots, V^{\rho_{X}}$ de courbes rationnelles irréductibles sur $X$ dont les classes dans $\mathrm{N}_{1}(X)_{\mathbb{Q}}$ sont linéairement indépendantes et des courbes $C^{1}, \ldots, C^{\rho_{X}}$ avec $\left[C^{j}\right] \in V^{j}$ pour tout $1 \leq j \leq \rho_{X}$ et $C^{j} \cap C^{j+1} \neq \varnothing$ pour tout $1 \leq j \leq \rho_{X}-1$, on a $\rho_{X}\left(\iota_{X}-1\right) \leq n$.

\section{Notations}

1.1. Si $X$ est une variété projective lisse, on note

$$
\mathrm{N}_{1}(X)_{\mathbb{Q}}=\left\{\sum_{i} a_{i} C_{i} \mid a_{i} \in \mathbb{Q}, C_{i} \text { courbe irréductible de } X\right\} / \equiv
$$

où $\equiv$ désigne l'équivalence numérique. Le cône de Mori, ou cône des courbes effectives, est le sous-cône de $\mathrm{N}_{1}(X)_{\mathbb{Q}}$ défini par

$$
\mathrm{NE}(X)=\left\{Z \in \mathrm{N}_{1}(X)_{\mathbb{Q}} \mid Z \equiv \sum_{i} a_{i} C_{i}, a_{i} \geq 0\right\} .
$$

Si $E$ est un diviseur et $R$ une arête de $\mathrm{NE}(X)$, la notation $E \cdot R>0$ (resp. $E \cdot R=0$ ) signifie $E \cdot C>0$ (resp. $E \cdot C=0$ ) pour toute courbe $C$ de $X$ telle que $[C] \in R$. La partie de $\mathrm{NE}(X)$ située dans le demi-espace ouvert $\left\{-K_{X}>0\right\}$ est localement polyédrale et pour toute arête $R$ de $\mathrm{NE}(X)$ telle que $-K_{X} \cdot R>0$, il existe un morphisme $\varphi_{R}: X \rightarrow X_{R}$ à fibres connexes, appelé contraction extrémale, de $X$ sur une variété projective normale $X_{R}$ tel que les courbes irréductibles contractées $\operatorname{par} \varphi_{R}$ sont exactement celles dont la classe dans $\mathrm{N}_{1}(X)_{\mathbb{Q}}$ appartient à $R$. 
- Soit $\operatorname{dim}\left(X_{R}\right)<\operatorname{dim}(X) ;$ on dit que $\varphi_{R}$ est une fibration, et $\rho_{X_{R}}=\rho_{X}-1$.

- Soit $\varphi_{R}$ est birationnelle et son lieu exceptionnel est un diviseur irréductible $E$ tel que $E \cdot R<0$ : on dit que $\varphi_{R}$ est une contraction divisorielle, et $\rho_{X_{R}}=\rho_{X}-1$.

- Soit $\varphi_{R}$ est birationnelle et son lieu exceptionnel est de codimension $>1$ dans $X$ : on dit que $\varphi_{R}$ est une petite contraction.

Si $X$ est une variété de Fano, le cône $\operatorname{NE}(X)$ est polyédral et si $\varphi_{R}: X \rightarrow X_{R}$ est une contraction extrémale, $\operatorname{NE}\left(X_{R}\right)$ est engendré par les $\varphi_{R}\left(R^{\prime}\right)$ où $R^{\prime}$ décrit l'ensemble (fini) des arêtes de $\mathrm{NE}(X)$.

1.2. Rappelons un résultat de Wiśniewski ([Wi91], Theorem (1.1)) sur le lieu d'une contraction extrémale. Soit $\varphi_{R}: X \rightarrow X_{R}$ une telle contraction, d'arête $R$. Notons $E_{R}$ le lieu de $X$ couvert par les courbes contractées par $\varphi_{R}$ et

$$
l(R)=\min \left\{-K_{X} \cdot C \mid C \text { courbe rationnelle contractée par } \varphi_{R}\right\}
$$

la longueur de $R$. Pour toute composante irréductible $F$ d'une fibre non triviale de $\varphi_{R}$, on a

$$
2 \operatorname{dim}\left(E_{R}\right) \geq \operatorname{dim}\left(E_{R}\right)+\operatorname{dim}(F) \geq \operatorname{dim}(X)+l(R)-1 \geq \operatorname{dim}(X)+\iota_{X}-1 .
$$

1.3. Nous utiliserons aussi le résultat suivant : soient $X$ une variété de Fano, $R$ une arête de $\mathrm{NE}(X)$ et $\varphi_{R}: X \rightarrow X_{R}$ la contraction associée. Si toutes les fibres de $\varphi_{R}$ sont de dimension $1, X_{R}$ est lisse ([An85], Theorem 3.1(ii)) et une fibre générale $F$ de $\varphi_{R}$ est une courbe rationnelle lisse qui vérifie $-K_{X} \cdot F=2 \geq \iota_{X}$. Deux cas sont alors possibles :

- soit $\varphi_{R}$ est une fibration en $\mathbb{P}^{1}$ et $X_{R}$ est une variété de Fano ([KMM92], Corollary 2.9);

- soit $\varphi_{R}$ a au moins une fibre singulière et $\iota_{X}=1$.

\section{La dimension trois}

2.1. Vérifions l'inégalité $(*)$ en dimension 3 . Le seul cas non trivial et non couvert par le résultat de Wiśniewski est donné par la proposition suivante.

Proposition 2.2. Soit $X$ une variété de Fano de dimension 3. Si $\iota_{X}=2$, on a $\rho_{X} \leq 3$.

Démonstration. Supposons $\rho_{X} \geq 4$. La variété $X$ possède au moins 4 contractions extrémales distinctes qui ne peuvent pas toutes être des fibrations ([Wi91], Theorem (2.2)). Les contractions extrémales sont complètement décrites par Mori ([Mo82], Theorem 3.3) et seule la contraction birationnelle lisse de centre un point est de longueur au moins 2. Cette possibilité est par exemple exclue par la classification des variétés dont l'éclatée en un point est de Fano ([BCW01]). 
Toujours en dimension 3, le cas d'égalité dans l'inégalité $(*)$ est caractérisé par la proposition suivante.

Proposition 2.3. Si $\rho_{X}\left(\iota_{X}-1\right)=\operatorname{dim}(X)=3$, on $a X \simeq\left(\mathbb{P}^{\iota_{X}-1}\right)^{\rho_{X}}$.

Démonstration. Si $\rho_{X}=1$ et $\iota_{X}=4$, la variété $X$ est isomorphe à $\mathbb{P}^{3}$ ([CMS00], $[\mathrm{Ke} 01])$. Supposons $\rho_{X}=3$ et $\iota_{X}=2$ et considérons une contraction extrémale $\varphi: X \rightarrow Z$. Si $\varphi$ est divisorielle de lieu exceptionnel $E$, alors $\varphi(E)$ est une courbe (cf. 1.2) et $\varphi$ s'identifie à l'éclatement d'une courbe lisse dans la variété lisse $Z$ ([Mo82], Corollary 3.4.1), ce qui est absurde puisque $\iota_{X}=2$. Les contractions extrémales sont donc toutes des fibrations et le résultat annoncé est donné par la proposition qui suit.

Proposition 2.4. Soit $X$ une variété de Fano de dimension $n$, de nombre de Picard $\geq n$ et de pseudo-indice $\geq 2$. Si toutes les contractions extrémales de $X$ sont des fibrations, $X$ est isomorphe à $\left(\mathbb{P}^{1}\right)^{n}$.

Démonstration. Montrons la proposition par récurrence sur $n$, le cas $n=1$ étant immédiat. Par [Wi91], Theorem (2.2), toutes les contractions extrémales sont de dimension relative 1 et il y en a exactement $n$ : le cône $\mathrm{NE}(X)$ est donc simplicial. Notons $R_{1}, \ldots, R_{n}$ ses arêtes. Pour $1 \leq i \leq n-1$, le cône $V_{i}=R_{1}+\cdots+R_{i}$ est une face extrémale de $\mathrm{NE}(X)$ dont on note $\varphi_{i}: X \rightarrow Z_{i}$ la contraction. Par le lemme de rigidité, $\varphi_{i+1}$ se factorise en $X \stackrel{\varphi_{i}}{\rightarrow} Z_{i} \rightarrow Z_{i+1}$. L'inclusion $V_{i} \subset V_{i+1}$ étant stricte, on a $\operatorname{dim}\left(Z_{i}\right)>\operatorname{dim}\left(Z_{i+1}\right)$. On en déduit $\operatorname{dim}\left(Z_{n-1}\right)=1$ et $Z_{n-1}$ est isomorphe à $\mathbb{P}^{1}$.

Pour $1 \leq i \leq n$, on considère la contraction $\varphi_{R_{i}}: X \rightarrow W_{i}$. Les fibres de $\varphi_{n-1}$ et de $\varphi_{R_{n}}$ se coupent en un nombre fini de points. Le morphisme $\varphi_{R_{n}}$ est donc équidimensionnel de dimension relative 1 . Il en résulte que $W_{n}$ est une variété de Fano lisse $(c f .1 .3)$ et que $\varphi_{R_{n}}$ est une fibration en $\mathbb{P}^{1}$ puisque $\iota_{X} \geq 2$. Comme toute arête de $\mathrm{NE}\left(W_{n}\right)$ est image d'une arête de $\mathrm{NE}(X)$, le cône $\mathrm{NE}\left(W_{n}\right)$ est simplicial et toute contraction extrémale de $W_{n}$ est une fibration.

Lemme 2.5. Soit $\pi: X \rightarrow Y$ une fibration en $\mathbb{P}^{r}$ entre variétés projectives et lisses. On suppose que $X$ est une variété de Fano. Alors,

(a) $Y$ est une variété de Fano et $\iota_{Y} \geq \iota_{X}$;

(b) si $Y$ vérifie $(*)$, il en est de même de $X$;

(c) si $\iota_{Y}=\iota_{X}$ et que $\mathbb{P}^{1} \rightarrow Y$ est une courbe de degré anticanonique $\iota_{Y}$, le produit $\mathbb{P}^{1} \times_{Y} X$ est isomorphe à $\mathbb{P}^{1} \times \mathbb{P}^{r}$.

Démonstration. Par [KMM92], Corollary 2.9, $Y$ est une variété de Fano. Soit $\mathbb{P}^{1} \rightarrow$ $Y$ une courbe de degré anticanonique $\iota_{Y}$. Comme $H^{2}\left(\mathbb{P}^{1}, \mathcal{O}_{\mathbb{P} 1}^{*}\right)$ est nul, il résulte de [Gr68], § 8, que la fibration $\mathbb{P}^{1} \times_{Y} X \rightarrow \mathbb{P}^{1}$ est isomorphe au projectifié d'un fibré vectoriel $E=\bigoplus_{i=0}^{r} \mathcal{O}_{\mathbb{P}^{1}}\left(a_{i}\right)$ sur $\mathbb{P}^{1}$, où les $a_{i}$ sont des entiers positifs avec 
$a_{0}=0$. Si $C_{i}$ est la section de $\mathbb{P}(E) \rightarrow \mathbb{P}^{1}$ définie par le fibré quotient $\mathcal{O}_{\mathbb{P}^{1}}\left(a_{i}\right)$ de $E$, on a $-K_{\mathbb{P}(E) / \mathbb{P}^{1}} \cdot C_{i}=-r a_{i}$, d'où, en notant $g$ la composée $\mathbb{P}^{1} \rightarrow C_{i} \rightarrow X$,

$$
\begin{aligned}
\iota_{X} \leq-K_{X} \cdot g_{*} C_{i} & =-\pi^{*} K_{Y} \cdot g_{*} C_{i}-K_{X / Y} \cdot g_{*} C_{i}=\iota_{Y}-g^{*} K_{X / Y} \cdot C_{i} \\
& =\iota_{Y}-K_{\mathbb{P}(E) / \mathbb{P}^{1}} \cdot C_{i}=\iota_{Y}-r a_{i}
\end{aligned}
$$

ce qui prouve (a) et (c).

Montrons (b). On a $\rho_{Y}=\rho_{X}-1$, d'où, en utilisant (a),

$$
\left(\rho_{X}-1\right)\left(\iota_{X}-1\right) \leq \rho_{Y}\left(\iota_{Y}-1\right) \leq \operatorname{dim}(Y)=\operatorname{dim} X-r .
$$

Si $C$ est une droite contenue dans une fibre de $\pi$, on a $-K_{X} \cdot C=r+1$, de sorte que $\iota_{X} \leq r+1$ et

$$
\rho_{X}\left(\iota_{X}-1\right) \leq \iota_{X}-1+\operatorname{dim}(X)-r \leq \operatorname{dim}(X),
$$

ce qui prouve (b).

On a donc $\iota_{W_{n}} \geq 2$ et par hypothèse de récurrence, $W_{n}$ est isomorphe à $\left(\mathbb{P}^{1}\right)^{n-1}$. De plus, l'image réciproque de toute droite du type $\mathbb{P}^{1} \times\left\{\left(t_{2}, \ldots, t_{n-1}\right)\right\}$ est isomorphe à $\left(\mathbb{P}^{1}\right)^{2}$. Puisque $\mathrm{NE}(X)$ est simplicial, la contraction $\varphi_{R_{1}+R_{n}}$ est la composée de $\varphi_{R_{n}}$ et d'une projection $p: W_{n} \rightarrow\left(\mathbb{P}^{1}\right)^{n-2}$; de même, $\varphi_{n-1}$ est la composée de $\varphi_{R_{n}}$ et d'une projection $W_{n} \rightarrow \mathbb{P}^{1}$. On a ainsi un diagramme commutatif

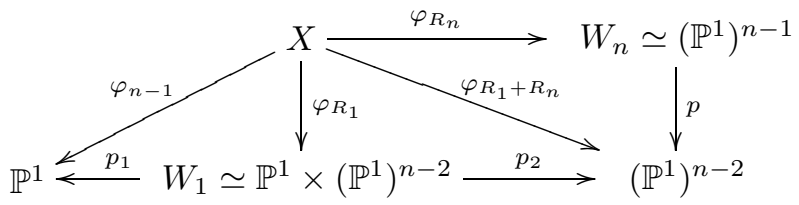

où $p_{1}$ et $p_{2}$ sont les deux projections. L'image réciproque d'un point de $\left(\mathbb{P}^{1}\right)^{n-2}$ par $\varphi_{R_{1}+R_{n}}$ est isomorphe à $\left(\mathbb{P}^{1}\right)^{2}$, les restrictions de $\varphi_{R_{1}}$ et $\varphi_{R_{n}}$ étant les projections sur chacun des facteurs. En d'autres termes, une fibre de $\varphi_{R_{n}}$ est envoyée isomorphiquement par $\varphi_{R_{1}}$ sur le facteur $\mathbb{P}^{1}$ de $W_{1}$. Cela signifie que le degré du morphisme produit

$$
\varphi_{n-1} \times \varphi_{R_{n}}: X \rightarrow \mathbb{P}^{1} \times\left(\mathbb{P}^{1}\right)^{n-1},
$$

qui est le degré de la restriction de $\varphi_{n-1}$ à une fibre générale de $\varphi_{R_{n}}$, vaut 1 . Ce morphisme étant fini, c'est un isomorphisme et la preuve de la proposition est achevée.

\section{La dimension quatre}

3.1. Montrons l'inégalité $(*)$ en dimension 4 . Une remarque essentielle est que sur une variété de Fano de dimension 4 et de pseudo-indice $\geq 2$, il n'y a pas de petite contraction (cf. 1.2). 
Proposition 3.2. Soit $X$ une variété de Fano de dimension 4. Si $\iota_{X}=3$, on a $\rho_{X} \leq 2$.

Démonstration. Supposons $\rho_{X} \geq 3$. Les fibrations extrémales sont de dimension relative au moins $2(c f .1 .2)$ et il y en a donc au plus 2 ([Wi91], Theorem (2.2)). Il existe ainsi au moins une contraction divisorielle contractant son lieu exceptionnel sur un point $(c f .1 .2)$. Il en résulte l'existence sur $X$ d'une contraction lisse de centre de codimension 2 ([Wi91], Corollary (1.3)), ce qui est absurde puisqu'une telle contraction est de longueur 1.

Le résultat principal de cette section est le théorème suivant.

Théorème 3.3. Soit $X$ une variété de Fano de dimension 4 . Si $\iota_{X}=2$, on a $\rho_{X} \leq 4$.

3.4. Quelques lemmes intermédiaires. La démonstration du théorème 3.3 repose sur les lemmes suivants.

Lemme 3.5. Une variété de Fano $X$ de dimension 4, de pseudo-indice 2 et de nombre de Picard $\geq 5$ n'a pas de fibration extrémale $\varphi: X \rightarrow Y$ dont toutes les fibres sont de dimension 1.

Démonstration. Raisonnons par l'absurde. Le morphisme $\varphi: X \rightarrow Y$ est une fibration lisse car $\iota_{X}=2$, de sorte que $Y$ est une variété de Fano lisse de dimension 3 d'après 1.3. Puisque $\rho_{Y} \geq 4$, il existe une contraction lisse $\pi: Y \rightarrow Z$ de centre une courbe lisse ([MM81], Theorem 5). Toute courbe de $Y$ contractée par $\pi$ est une courbe rationnelle de fibré normal dans $Y$ égal à $\mathcal{O}(-1) \oplus \mathcal{O}$, donc de degré anticanonique 1 . Ceci, avec le lemme 2.5, contredit l'hypothèse $\iota_{X}=2$.

Lemme 3.6. Soit $X$ une variété de Fano de dimension 4, de pseudo-indice 2 et de nombre de Picard $\geq 5$. Toute contraction extrémale $X \rightarrow Y$ est ou bien une contraction divisorielle lisse de centre une courbe, autrement dit est l'éclatement d'une variété lisse $Y$ le long d'une courbe lisse de $Y$, ou bien une fibration de dimension relative 1 .

Dans le cas d'une fibration, le lemme 3.5 montre qu'il y a au moins une fibre de dimension 2 .

Démonstration. Remarquons tout d'abord que les fibrations extrémales $X \rightarrow Y$ vérifient toutes $\operatorname{dim}(Y)=2$ ou $\operatorname{dim}(Y)=3$. En effet, si $\operatorname{dim}(Y)=0$ (resp. $\operatorname{dim}(Y)=1$ ), on a $\rho_{X}=1$ (resp. $\rho_{X}=2$ ). D'autre part, comme $\operatorname{dim}(X)=4$ et $\rho_{X} \geq 5$, il y a au moins une contraction extrémale divisorielle ([Wi91], Theorem $(2.2))$. 
Soient $\varphi: X \rightarrow Y$ une telle contraction et $E$ son diviseur exceptionnel. Les fibres non triviales de $\varphi$ sont de dimension $\geq 2$ (cf. 1.2). Si $\operatorname{dim}(\varphi(E))=0$, il existe sur $X$ une fibration extrémale dont toutes les fibres sont de dimension 1 ou une contraction extrémale birationnelle lisse de centre de codimension 2 ([Wi91], Corollary (1.3)), ces deux situations étant exclues respectivement par le lemme 3.5 et par l'hypothèse $\iota_{X}=2$. Ainsi, $\operatorname{dim}(\varphi(E))=1$ et $\varphi$ s'identifie à l'éclatement d'une variété lisse $Y$ le long de la courbe lisse $\varphi(E)$ ([AW98], Theorem 4.1).

Vérifions enfin qu'il n'existe pas de fibration extrémale $\pi: X \rightarrow S$ où $S$ est une surface. Supposons qu'une telle fibration existe; $S$ est alors une surface lisse ([ABW92], Proposition 1.4.1) et une fibre non triviale $F$ de $\varphi$ est isomorphe à $\mathbb{P}^{2}$. Puisque $\varphi_{\mid F}: F \rightarrow S$ est fini, $S$ est également isomorphe à $\mathbb{P}^{2}$, de sorte que $\rho_{X}=2$, ce qui est absurde.

Le résultat suivant précise le cas des éclatements de centre une courbe lisse.

Proposition 3.7. Soit $X$ une variété de Fano de dimension $n \geq 4$ et de pseudoindice $\iota_{X} \geq 2$. Si $\pi: X \rightarrow Y$ est l'éclatement d'une variété lisse $Y$ le long d'une courbe lisse, $Y$ est une variété de Fano et $\iota_{Y} \geq \iota_{X}$.

Démonstration. Si $Y$ n'est pas une variété de Fano, le centre de l'éclatement $\pi$ est une courbe rationnelle lisse de fibré normal $\mathcal{O}_{\mathbb{P}^{1}}(-1)^{\oplus n-1}$ ([Wi91], Proposition (3.5)), de sorte que $\iota_{X}=1$, ce qui contredit l'hypothèse.

Vérifions ensuite l'inégalité $\iota_{Y} \geq 2$. Soient $C$ le centre de $\pi$ et $E$ son diviseur exceptionnel. Si $C^{\prime}$ est une courbe rationnelle de $Y$ distincte de $C$, et si on note encore $C^{\prime}$ sa transformée stricte dans $X$, on a $-K_{Y} \cdot C^{\prime}=-K_{X} \cdot C^{\prime}+(n-2) E \cdot C^{\prime} \geq$ $\iota_{X}$. Si $\iota_{Y}<\iota_{X}$, on ne peut donc avoir $-K_{Y} \cdot C^{\prime}=\iota_{Y}$, de sorte que $C$ est une courbe rationnelle qui satisfait $-K_{Y} \cdot C=\iota_{Y}$.

Soit $N_{C / Y}=\bigoplus_{i=1}^{n-1} \mathcal{O}_{\mathbb{P}^{1}}\left(a_{i}\right)$ son fibré normal. On a d'une part

$$
\iota_{Y}=-K_{Y} \cdot C=\sum_{i=1}^{n-1} a_{i}+2,
$$

et d'autre part, si $C_{i} \subset E$ est la courbe définie par le fibré quotient $\mathcal{O}_{\mathbb{P}^{1}}\left(a_{i}\right)$ de $N_{C / Y}$,

$$
-K_{X} \cdot C_{i}=\iota_{Y}-(n-2) a_{i} \geq \iota_{X}
$$

On en déduit $\iota_{Y} \geq \iota_{X}$, sauf si tous les $a_{i}$ sont strictement négatifs, ce qu'exclut l'égalité $\sum_{i=1}^{n-1} a_{i}=\iota_{Y}-2 \geq-1$.

3.8. Variétés de Fano spéciales. Nous nous intéressons ici à certaines variétés de Fano, que nous appelons «spéciales », faute d'une meilleure terminologie. Cette notion est introduite dans le seul but d'alléger les énoncés qui la suivent.

Définition. Une variété de Fano de dimension $n$ sera dite spéciale si toutes ses contractions extrémales sont ou bien des éclatements lisses de centre une courbe 
lisse ou bien des fibrations de dimension relative 1 et si elle possède au moins une contraction extrémale birationnelle.

Exemple. La variété $X=\mathbb{P}_{a}^{n} \times \mathbb{P}^{1}$, où $\mathbb{P}_{a}^{n}$ désigne l'éclatement de $\mathbb{P}^{n}$ de centre $a$, est une variété de Fano spéciale, de pseudo-indice 2 et de nombre de Picard 3. En effet, $X$ possède 3 contractions extrémales : $X \rightarrow \mathbb{P}_{a}^{n}$ et $X \rightarrow \mathbb{P}^{n-1} \times \mathbb{P}^{1}$, fibrations en $\mathbb{P}^{1}$, et $X \rightarrow \mathbb{P}^{n} \times \mathbb{P}^{1}$, contraction lisse de centre $\{a\} \times \mathbb{P}^{1}$.

Cet exemple est d'une certaine façon extrémal comme le montre le résultat suivant.

Théorème 3.9. Une variété de Fano spéciale $X$ de dimension $\geq 4$ vérifie $\rho_{X} \leq 3$.

La démonstration de ce résultat occupe la fin de ce paragraphe.

Lemme 3.10. Soit $X$ une variété de Fano spéciale de dimension $\geq 4$ et de nombre de Picard $\geq 4$.

(a) La variété $X$ possède au plus une fibration extrémale de dimension relative 1.

(b) Si $X$ possède une fibration extrémale de dimension relative 1 , d'arête $R$, les contractions extrémales birationnelles ont pour centre une courbe rationnelle à fibré normal trivial, leur diviseur exceptionnel $E$ vérifie $E \cdot R=0$ et $\iota_{X} \leq 2$.

Démonstration. Soient $\pi: X \rightarrow W$ une contraction extrémale birationnelle de centre une courbe lisse $C \subset W$ et de diviseur exceptionnel $E$ et $\varphi: X \rightarrow Y$ une fibration extrémale d'arête $R$ et de dimension relative 1 . Nous allons montrer que $\pi$ détermine $R$, donc $\varphi$.

Le morphisme $\varphi_{\mid E}: E \rightarrow Y$ n'est pas surjectif car $\rho_{E}=2$ et $\rho_{Y} \geq 3$; comme l'intersection d'une fibre de $\varphi_{\mid E}$ avec une fibre de $\pi_{\mid E}$ est finie, chaque fibre de $\varphi_{\mid E}$ est de dimension 1. En particulier, $\varphi(E)$ est de codimension 1 dans $Y$; l'image réciproque par $\varphi$ d'un point général de $\varphi(E)$ est de dimension 1 , donc coïncide avec son image réciproque par $\varphi_{\mid E}$. C'est en particulier une courbe rationnelle qui domine $C$, de sorte que cette dernière aussi est rationnelle.

Le diviseur $E$ est isomorphe à $\mathbb{P}\left(N_{C / W}^{*}\right)$, où $N_{C / W}=\bigoplus_{i=1}^{n-1} \mathcal{O}_{\mathbb{P}^{1}}\left(a_{i}\right)$. La fibration induite $\widetilde{\varphi}_{\mid E}: E \rightarrow \widetilde{\varphi}(E)$, où $\widetilde{\varphi}(E)$ est la normalisation de $\varphi(E)$, est équidimensionnelle, de fibre générale $\mathbb{P}^{1}$; c'est une contraction extrémale puisque $\rho_{E}=2$. Le cône de Mori de $\mathbb{P}\left(N_{C / W}^{*}\right)$ est engendré par deux courbes : une droite d'une fibre de $\pi_{\mid E}: E \rightarrow C$ et une section de $\pi_{\mid E}$. Il s'ensuit que $E$ est isomorphe à $\mathbb{P}^{n-2} \times \mathbb{P}^{1}$, que $\pi_{\mid E}$ et $\varphi_{\mid E}$ sont les deux projections et que $R$ est engendré par une courbe du type $\{*\} \times \mathbb{P}^{1}$. Comme $n \geq 4$, il y a donc au plus une fibration extrémale de dimension relative 1 . 
Ce qui précède montre aussi que si $X$ possède une fibration extrémale de dimension relative 1 , les contractions extrémales birationnelles ont pour centre une courbe rationnelle de fibré normal trivial. En effet, on a vu que $\mathbb{P}\left(N_{C / W}^{*}\right)$ est isomorphe à $\mathbb{P}^{n-2} \times \mathbb{P}^{1}$, de sorte que $N_{C / W}$ est isomorphe à $\mathcal{O}_{\mathbb{P}^{1}}(a)^{\oplus n-1}$ pour un entier $a$ convenable et, puisque les courbes du type $\{*\} \times \mathbb{P}^{1}$ ont un fibré normal (dans $X$ ) trivial (ce sont les fibres d'une fibration), c'est que $a=0$.

Lemme 3.11. Soit $X$ une variété projective lisse de dimension 4 et soit $\pi: X \rightarrow$ $W$ (resp. $\left.\pi^{\prime}: X \rightarrow W^{\prime}, \pi^{\prime \prime}: X \rightarrow W^{\prime \prime}\right)$ une contraction extrémale birationnelle lisse de centre une courbe lisse $C \subset W$ (resp. $\left.C^{\prime} \subset W^{\prime}, C^{\prime \prime} \subset W^{\prime \prime}\right)$ d'arête $R$ (resp. $\left.R^{\prime}, R^{\prime \prime}\right)$ et de diviseur exceptionnel $E$ (resp. $\left.E^{\prime}, E^{\prime \prime}\right)$.

(a) Si $R \neq R^{\prime}$ et $E \cap E^{\prime} \neq \varnothing$, on a $E \cdot R^{\prime}>0$ et $E^{\prime} \cdot R>0$.

(b) Si $R, R^{\prime}$ et $R^{\prime \prime}$ sont deux à deux distinctes et si $E \cap E^{\prime} \neq \varnothing$ et $E \cap E^{\prime \prime} \neq \varnothing$, on a $E^{\prime} \cap E^{\prime \prime} \neq \varnothing$.

Démonstration. Vérifions le point (a). Soit $x$ un point de $E \cap E^{\prime}$. La surface $\pi^{-1}(\pi(x))$, isomorphe à $\mathbb{P}^{2}$, n'est pas contenue dans $E^{\prime}$ car $R \neq R^{\prime}$. Il existe donc une courbe dans $\pi^{-1}(\pi(x))$ passant par $p$ et non contenue dans $E^{\prime}$, d'où (a) en échangeant les rôles de $E$ et $E^{\prime}$.

Vérifions le point (b). Soit $y \in C$. Les courbes $\pi^{-1}(y) \cap E^{\prime}$ et $\pi^{-1}(y) \cap E^{\prime \prime}$ sont contenues dans $\pi^{-1}(y)$, qui est isomorphe à $\mathbb{P}^{2}$, de sorte que $\pi^{-1}(y) \cap E^{\prime} \cap E^{\prime \prime} \neq \varnothing$, d'où (b).

3.12. Démonstration $\mathbf{d u}$ théorème 3.9. Supposons $\rho_{X} \geq 4$. Par hypothèse, il existe une contraction extrémale birationnelle $\pi: X \rightarrow W$ d'arête $R_{1}$ et de diviseur exceptionnel $E_{1}$, d'image une variété de Fano lisse (proposition 3.7 ) et de centre une courbe lisse $C \subset W$.

Soit $C_{1}$ une courbe telle que $E_{1} \cdot C_{1}>0$. Cette courbe est numériquement combinaison linéaire à coefficients rationnels strictement positifs de classes engendrant des arêtes. L'une d'entre elles, notée $R_{2}$, satisfait $E_{1} \cdot R_{2}>0$. Le lemme 3.10(b) entraîne que la contraction $\varphi_{R_{2}}$ est birationnelle.

Notons $R_{1}, R_{2}, \ldots, R_{k}, R_{k+1}, \ldots, R_{m}, R_{F}$ les arêtes de $\mathrm{NE}(X)$, où

- les contractions $\varphi_{R_{i}}$ sont birationnelles de diviseur exceptionnel $E_{i}$ (noter que $\left.\pi=\varphi_{R_{1}}\right)$;

- $\varphi_{R_{F}}$ est l'éventuelle unique fibration et, d'après le lemme 3.10 (b), $E_{i} \cdot R_{F}=0$ pour tout $1 \leq i \leq m$;

- $E_{1} \cap E_{i} \neq \varnothing$ pour $2 \leq i \leq k$ d'où, d'après le lemme 3.11(a), $E_{1} \cdot R_{i}>0$;

- $E_{1} \cap E_{i}=\varnothing$ pour $k+1 \leq i \leq m$, d'où, d'après le lemme 3.11(b), $E_{2} \cap E_{i}=\varnothing$.

Supposons $n \geq 5$. L'intersection $E_{1} \cap E_{2}$ n'est pas vide, de dimension au moins $n-2 \geq 3$ : il y a donc des courbes de $E_{1} \cap E_{2}$ contractées à la fois par $\varphi_{R_{1}}$ et par $\varphi_{R_{2}}$, ce qui est absurde. 
Supposons $n=4$ et vérifions que $C$ est extrémale dans $\mathrm{NE}(W)$. Rappelons que ce cône est engendré par $\pi\left(R_{F}\right)$ et les $\pi\left(R_{i}\right)$ pour $i \geq 2$. Pour $2 \leq i \leq k$, la classe de $C$ appartient à $\pi\left(R_{i}\right)$, ainsi qu'à $\pi\left(R_{F}\right)$ : en effet, chaque fibre non triviale de $\varphi_{R_{i}}$ intersecte $E_{1}$ le long d'une courbe $C_{i}$ telle que $\pi\left(C_{i}\right)=C$, de même, toute fibre $F$ de $\varphi_{R_{F}}$ intersectant $E_{1}$ est contenue dans $E_{1}$ et satisfait donc $\pi(F)=C$. Si la classe de $C$ n'est pas extrémale, $\operatorname{NE}(W)$ est donc engendré par les arêtes $\pi\left(R_{i}\right)$ pour $i \geq k+1$. L'image $\pi\left(E_{2}\right)$ est un diviseur effectif de $W$ numériquement trivial puisque $E_{2} \cdot R_{F}=E_{2} \cdot R_{i}=0$, ce qui est absurde.

Étudions la contraction extrémale $\varphi: W \rightarrow Y$ d'arête engendrée par $C$ et montrons que le diviseur $\pi\left(E_{2}\right)$ est contracté sur un point par $\varphi$. Notons $W_{2}$ l'image de $\varphi_{R_{2}}$ et $C_{2} \subset W_{2}$ son centre. Pour tout $y \in C_{2}$, la fibre $\varphi_{R_{2}}^{-1}(y)$, isomorphe à $\mathbb{P}^{2}$, contient une courbe dont l'image par $\pi$ est $C$ et qui est donc contractée par $\varphi$ sur le point $\varphi(C)$. D'autre part, $\pi\left(\varphi_{R_{2}}^{-1}(y)\right)$ est également contracté sur le point $\varphi(C)$. La contraction $\varphi: W \rightarrow Y$ est donc ou bien divisorielle ou bien une fibration. Soit $C^{\prime} \in R_{2}$ une courbe rationnelle de $X$ telle que $E_{2} \cdot C^{\prime}=-1$. Il existe un entier $r \geq 1$ tel que

$$
\pi\left(E_{2}\right) \cdot \pi_{*}\left(C^{\prime}\right)=\pi^{*}\left(\pi\left(E_{2}\right)\right) \cdot C^{\prime}=\left(E_{2}+r E\right) \cdot C^{\prime}=-1+r E \cdot C^{\prime} .
$$

Or $E \cdot C^{\prime} \geq 1$; on en déduit $\pi\left(E_{2}\right) \cdot \pi_{*}\left(C^{\prime}\right) \geq 0$ puis, puisque les courbes $C$ et $\pi_{*}\left(C^{\prime}\right)$ sont numériquement proportionnelles, $\pi\left(E_{2}\right) \cdot C \geq 0$.

Comme $\pi\left(E_{2}\right)$ est contracté sur un point par $\varphi$, le calcul d'intersection précédent montre que $\varphi$ est une fibration. En particulier, $\operatorname{dim}(Y) \leq 1$ et $\rho_{W} \leq 2$, ce qui est absurde puisqu'on a supposé $\rho_{X} \geq 4$.

3.13. Démonstration du théorème 3.3. Le lemme 3.6 montre que si $X$ est une variété de Fano de dimension 4, de pseudo-indice 2 et de nombre de Picard $\geq 5$, la contraction extrémale associée à toute arête extrémale de $X$ est soit une contraction divisorielle lisse de centre une courbe lisse, soit une fibration de dimension relative 1. De plus, comme $\rho_{X} \geq 5$, il existe sur $X$ au moins une contraction extrémale birationnelle ([Wi91], Theorem (2.2)). C'est donc que $X$ est spéciale et le théorème 3.9 permet de conclure à une absurdité.

3.14. Familles propres de courbes rationnelles. Nous renvoyons au livre [Ko96] pour plus de détails sur les notations et les rappels qui suivent. Soit $X$ une variété complexe, projective et lisse. Soit $\operatorname{Hom}_{\text {bir }}\left(\mathbb{P}^{1}, X\right)$ le schéma des morphismes birationnels de $\mathbb{P}^{1}$ vers $X$ et soit $\operatorname{Hom}_{\text {bir }}^{n}\left(\mathbb{P}^{1}, X\right)$ sa normalisation. Le groupe linéaire $\operatorname{PGL}(2, \mathbb{C})$ agit sur $\operatorname{Hom}_{\text {bir }}^{n}\left(\mathbb{P}^{1}, X\right)$ et $\operatorname{Hom}_{\text {bir }}^{n}\left(\mathbb{P}^{1}, X\right) \times \mathbb{P}^{1}$. Les quotients géométriques au sens de Mumford existent et seront respectivement notés RatCurves ${ }^{n}(X)$ et $\operatorname{Univ}^{r c}(X)$. Soit $V \subset \operatorname{RatCurves}^{n}(X)$ une famille propre irréductible de courbes rationnelles irréductibles sur $X$ et soit $\mathcal{U} \subset \operatorname{Univ}^{r c}(X)$ la 
famille universelle

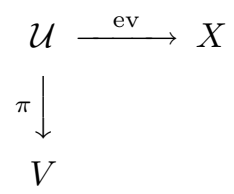

Notons lieu $(V)=\operatorname{ev}(\mathcal{U})$ l'ensemble des points de $X$ par lesquels il passe une courbe rationnelle $C$ de $X$ telle que $[C] \in V$. Soit $x \in \operatorname{lieu}(V)$; on note $V_{x}=\pi\left(\mathrm{ev}^{-1}(x)\right) \subset$ $V$ les courbes de $V$ passant par $x$, puis $\mathcal{U}_{x}=\pi^{-1}\left(V_{x}\right)$ et $\operatorname{lieu}\left(V_{x}\right)=\operatorname{ev}\left(\mathcal{U}_{x}\right)$. Les dimensions de ces différentes variétés satisfont

$$
\begin{aligned}
\operatorname{dim}(V) & \geq-K_{X} \cdot V+n-3 \\
\operatorname{dim}\left(V_{x}\right) & \geq-K_{X} \cdot V-2
\end{aligned}
$$

où $-K_{X} \cdot V$ désigne l'intersection $-K_{X} \cdot C$ pour une courbe rationnelle $C$ de $X$ telle que $[C] \in V$. Si $x$ est un point général de lieu $(V)$, on a

$$
\operatorname{dim}(\operatorname{lieu}(V))+\operatorname{dim}\left(V_{x}\right)=\operatorname{dim}(V)+1
$$

et, par le lemme de cassage ([Mo79], Theorem 6),

$$
\operatorname{dim}\left(\operatorname{lieu}\left(V_{x}\right)\right)=\operatorname{dim}\left(V_{x}\right)+1 \geq-K_{X} \cdot V-1 .
$$

Nous utiliserons de façon répétée le résultat suivant ([Ko96], II.4.21).

Lemme 3.15. Soit $V \subset$ RatCurves $^{n}(X)$ une famille propre et irréductible de courbes rationnelles irréductibles sur une variété $X$ projective et lisse et soit $x \in$ lieu $(V)$. Toute courbe tracée sur lieu $\left(V_{x}\right)$ est numériquement proportionnelle à une courbe $C$ telle que $[C] \in V$.

3.16. La famille $V$ détermine une relation d'équivalence sur $X$ pour laquelle des points $x$ et $x^{\prime}$ de $X$ sont équivalents s'il existe une chaîne connexe de courbes rationnelles de $V$ passant par $x$ et $x^{\prime}$. Il existe un ouvert $X_{0} \subset X$ et un morphisme propre $X_{0} \rightarrow Z_{0}$ à fibres connexes vers une variété normale dont les fibres sont des classes d'équivalence pour la relation précédente ([Ko96], IV.4.16).

3.17. Les cas d'égalité. L'objet de ce paragraphe est l'étude des cas d'égalité dans $(*)$.

Proposition 3.18. Soit $X$ une variété de Fano de dimension 4 . Si $\rho_{X}\left(\iota_{X}-1\right)=4$, la variété $X$ est isomorphe à $\left(\mathbb{P}^{\iota-1}\right)^{\rho_{X}}$.

Démonstration. Si $\rho_{X}=1$ et $\iota_{X}=5$, la variété $X$ est isomorphe à $\mathbb{P}^{4}$ ([CMS00], $[\mathrm{Ke} 01])$.

Supposons $\rho_{X}=2$ et $\iota_{X}=3$. Notons $\varphi_{1}: X \rightarrow W_{1}$ et $\varphi_{2}: X \rightarrow W_{2}$ les deux contractions extrémales, d'arêtes respectives $R_{1}$ et $R_{2}$. Elles sont ou bien 
divisorielles ou bien des fibrations (cf. 1.2). Supposons par exemple que $\varphi_{1}$ soit divisorielle. Le lieu exceptionnel $E_{1}$ de $\varphi_{1}$ est contracté sur un point par $\varphi_{1}$ (cf. 1.2). Rappelons que l'intersection de fibres de deux contractions extrémales différentes est finie. Les fibres non triviales de $\varphi_{2}$ sont de dimension au moins 2 , donc ne rencontrent pas $E_{1}$, autrement dit, $\varphi_{2}$ est également divisorielle de lieu exceptionnel $E_{2}$ disjoint de $E_{1}$. Le diviseur $-E_{1}$ est donc numériquement effectif puisque $-E_{1} \cdot R_{1}>0$ et $-E_{1} \cdot R_{2}=0$, ce qui est manifestement absurde. Les morphismes $\varphi_{1}$ et $\varphi_{2}$ sont donc des fibrations équidimensionnelles de dimension relative 2 ( $c f$. 1.2 ) et $W_{1}$ et $W_{2}$ sont lisses ([ABW92], Proposition 1.4.1). Une fibre générale $F_{1}$ (resp. $F_{2}$ ) de $\varphi_{1}$ (resp. $\varphi_{2}$ ) est de pseudo-indice 3 par la formule d'adjonction et donc isomorphe à $\mathbb{P}^{2}$. Le morphisme $F_{1} \rightarrow W_{2}$ (resp. $F_{2} \rightarrow W_{1}$ ) est fini et $W_{1}$ (resp. $\left.W_{2}\right)$ est isomorphe à $\mathbb{P}^{2}$ par le théorème de Lazarsfeld ([La83]).

Les fibres de $\varphi_{2}$ ne sont a priori pas des sections de $\varphi_{1}$. Nous allons montrer que de telles sections existent. Si $\ell \subset \mathbb{P}^{2}$ est une droite générale, $X_{\ell}=\varphi_{1}^{-1}(\ell)$ est lisse et connexe. Les fibres générales de $X_{\ell} \rightarrow \ell$ sont isomorphes à $\mathbb{P}^{2}$. Il existe donc une section de $X_{\ell} \rightarrow \ell$ par le théorème de Tsen ([Ko96], Theorem IV.6.5).

Soit donc $C \subset X$ une courbe rationnelle vérifiant $C \cdot \varphi_{1}^{*} \mathcal{O}_{\mathbb{P}^{2}}(1)=1$, de degré minimal relativement au diviseur ample $-K_{X}$. La courbe $C$ détermine une famille propre irréductible $V \subset$ RatCurves $^{n}(X)$ de courbes rationnelles irréductibles sur $X$ (cf. 3.14). La dimension de $V$ est au moins $-K_{X} \cdot C+1 \geq 4$ et celle de $V_{x}$, pour $x \in \operatorname{lieu}(V)$, au moins $-K_{X} \cdot C-2 \geq 1$. Si la dimension de lieu $\left(V_{x}\right)$ est au moins 3 , il rencontre une fibre générale de $\varphi_{1}$ au moins le long d'une courbe et $[C] \in R_{1}$ ( $c f$. lemme 3.15 ) ce qui est absurde par le choix de $C$. Ainsi, $\operatorname{dim}\left(\operatorname{lieu}\left(V_{x}\right)\right)=2$ et le lieu de $V$ est $X$ par la formule (2) de 3.14 .

Il existe un ouvert $X_{0} \subset X$, une variété normale $Z_{0}$ et un morphisme propre $q: X_{0} \rightarrow Z_{0}$ à fibres connexes dont les fibres sont des classes pour la relation d'équivalence déterminée par $V(c f .3 .14)$. La famille $V$ est couvrante et $Z_{0}$ est donc de dimension au plus 3. Soient $F$ une fibre générale de $q$ et $x$ un point de $F$. Comme $\operatorname{dim}\left(\operatorname{lieu}\left(V_{x}\right)\right)=2$, on a $\operatorname{dim}(F) \geq 2$. Si $F$ est de dimension au moins 3 , elle rencontre une fibre générale de $\varphi_{1}$ au moins le long d'une courbe. Comme $F$ est rationnellement connexe relativement à une sous-famille propre et irréductible de courbes de $V$, toutes les courbes de $F$ sont algébriquement équivalentes à un multiple de $C$ ([Ko96], IV 3.13.3) et $[C] \in R_{1}$, ce qui est à nouveau absurde. Ainsi $F$ est une surface de pseudo-indice 3 par la formule d'adjonction, donc isomorphe à $\mathbb{P}^{2}$; on a $F=\operatorname{lieu}\left(V_{x}\right)$ pour tout $x$ dans $F$, les courbes de $V_{x}$ étant les droites passant par $x$. Notons que $F$ est une section de $\varphi_{1}$.

Vérifions que l'on peut prendre $X_{0}=X$. Le fibré normal à $F$ dans $X$ est trivial et le schéma de Hilbert de $X$ est donc lisse au point $[F]$. Soient $H$ son unique composante passant par $[F]$ et $X^{\prime} \subset X \times H$ la famille universelle. Quitte à remplacer $Z_{0}$ par un ouvert dense, on peut supposer que $q$ est plat et on a un 
diagramme

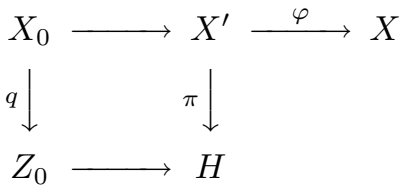

où le carré est cartésien et où la composée des flèches horizontales supérieures est l'inclusion. Le morphisme $\pi$ s'identifie, au-dessus d'un ouvert $H_{0}$ convenable de $H$, au morphisme $X_{0} \rightarrow Z_{0}$.

Fixons un point $t_{0}$ de $H$ et des points $x_{1}^{\prime}$ et $x_{2}^{\prime}$ de $\pi^{-1}\left(t_{0}\right)$. Notons $T \rightarrow H$ un germe de courbe lisse passant par $t_{0}$ et rencontrant $H_{0}$. Le schéma $\pi_{T}: X_{T}^{\prime} \rightarrow T$ obtenu par le changement de base $T \rightarrow H$ est irréductible : il existe donc des courbes $T_{1} \rightarrow X_{T}^{\prime}$ et $T_{2} \rightarrow X_{T}^{\prime}$ dominant $T$ et rencontrant $\pi_{T}^{-1}\left(t_{0}\right)$ uniquement en $x_{1}^{\prime}$ et $x_{2}^{\prime}$ respectivement. Pour tous points $t_{1} \in T_{1}$ et $t_{2} \in T_{2}$ situés au-dessus du même point général $t$ de $T$, les points correspondant de $X$ peuvent être joints par une (unique) courbe $C_{t_{1}, t_{2}} \subset \pi_{T}^{-1}(t)$ de $V$. Cette famille étant propre, les courbes $C_{t_{1}, t_{2}}$ dégénèrent vers une courbe de $V$, qui est en particulier irréductible, joignant $x_{1}^{\prime}$ et $x_{2}^{\prime}$. Les fibres de $\pi$ sont donc irréductibles et deux points quelconques sont reliés par une courbe de $V$.

Supposons que le morphisme $\varphi$ contracte une courbe irréductible $C \subset X^{\prime}$ vers un point $x$ de $X$. Celle-ci est horizontale pour $\pi$ et le lieu couvert par les $\varphi\left(X_{t}^{\prime}\right)$, pour $t \in \pi(C)$, est de dimension 3. Ainsi, le lieu couvert par les courbes de $V_{x}$ est de dimension 3, ce qui est exclu par les arguments précédents. Le morphisme $\varphi$ est donc birationnel et fini : c'est un isomorphisme.

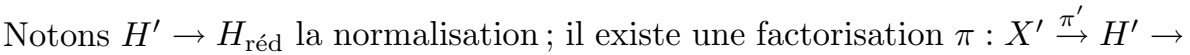
$H_{\text {réd }}$ et le morphisme $q^{\prime}=\pi^{\prime} \circ \varphi^{-1}: X \rightarrow H^{\prime}$ étend $q$. Le morphisme produit $\varphi_{1} \times q^{\prime}: X \rightarrow \mathbb{P}^{2} \times H^{\prime}$ est birationnel (puisqu'une fibre générale de $q^{\prime}$ est une section de $\varphi_{1}$ ); puisque $\rho_{X}=2$, il est fini : c'est un isomorphisme. On en déduit que $H^{\prime}$ est lisse et, comme les fibres de $\varphi_{1}$, isomorphe à $\mathbb{P}^{2}$.

Supposons $\rho_{X}=4$ et $\iota_{X}=2$. Par 1.2, toute contraction extrémale birationnelle est divisorielle et ses fibres non triviales sont de dimension $\geq 2$. Vérifions que les fibrations extrémales, s'il en existe, sont de dimension relative 1 . Soit $\varphi: X \rightarrow Z$ une telle contraction; supposons qu'elle soit de dimension relative au moins 2 . Notons que $Z$ est lisse ([ABW92], Proposition 1.4.1). Puisque $\rho_{X}=4$, il existe ([Wi91], Theorem (2.2)) une contraction extrémale birationnelle $\psi: X \rightarrow W$; ses fibres non triviales sont de dimension $\geq 2$, de sorte que $\varphi$ est de dimension relative 2. La variété $W$ est donc lisse et $\psi$ s'identifie à l'éclatement d'une courbe lisse dans $W$ ([AW98], Theorem 4.1). Toute fibre de $\psi_{\mid E}: E \rightarrow \psi(E)$ est en particulier isomorphe à $\mathbb{P}^{2}$ et domine $Z$. Il en résulte que $Z$ est isomorphe à $\mathbb{P}^{2}$ ([La83]) ce qui est absurde puisque $\rho_{Z}=\rho_{X}-1=3$.

La variété $X$ n'est pas spéciale (théorème 3.9) et ou bien toutes les contractions élémentaires sont des fibrations de dimension relative 1 , ou bien il existe une 
contraction birationnelle $\psi: X \rightarrow W$, divisorielle, dont le lieu exceptionnel $E$ est contracté sur un point. Vérifions que ce dernier cas ne peut pas se produire. Il existe alors une contraction extrémale $\varphi: X \rightarrow Z$ dont le lieu exceptionnel rencontre $E$. L'intersection d'une fibre de $\varphi$ avec $E$ étant finie, cette fibre est de dimension au plus 1. Puisque $\iota_{X}=2$, le morphisme $\varphi$ est une fibration lisse de dimension relative 1 par 1.3. On en déduit que $Z$ est une variété de Fano de dimension 3, de nombre de Picard 3 et de pseudo-indice au moins 2 (lemme $2.5)$ et donc exactement $2(c f$. section 2$)$. Finalement, $Z$ est isomorphe à $\left(\mathbb{P}^{1}\right)^{3}$ (proposition 2.3). Posons $\ell=\mathbb{P}^{1} \times\left\{\left(t, t^{\prime}\right)\right\}$, pour $t$ et $t^{\prime}$ généraux dans $\mathbb{P}^{1}$. La surface $X_{\ell}=\varphi^{-1}(\ell)$ est une surface de Hirzebruch et la courbe $\ell^{\prime}=E \cap X_{\ell}$ est exceptionnelle car contractée par $\psi:$ c'est donc une section de $\varphi_{\mid X_{\ell}}: X_{\ell} \rightarrow \ell$. Le morphisme $E \rightarrow Z$ est birationnel et fini : c'est un isomorphisme. On a $-K_{E} \cdot \ell^{\prime}=2$ et $-E \cdot \ell^{\prime}>0$ puisque $\ell^{\prime}$ est exceptionnelle dans $X_{\ell}$. Or, par la formule d'adjonction, on a $-K_{E} \cdot \ell^{\prime}=-K_{X} \cdot \ell^{\prime}-E \cdot \ell^{\prime}>2$ ce qui est absurde.

En conclusion, toutes les contractions extrémales de $X$ sont des fibrations. Le résultat cherché est donné par la proposition 2.4.

La démonstration précédente, où l'on remplace le théorème de Tsen par sa version générale récente due à Graber, Harris et Starr [GHS03], montre le résultat suivant.

Théorème 3.19. Soient $X$ une variété de Fano de dimension $n$ et $Z$ une variété lisse de dimension $m$ avec $\iota_{X} \geq m+1$. S'il existe un morphisme surjectif à fibres connexes $f: X \rightarrow Z$, alors $Z$ est isomorphe à $\mathbb{P}^{m}$ et il existe une variété lisse $Y$ de dimension $n-m$ telle que $X$ est isomorphe à $Y \times \mathbb{P}^{m}$.

\section{Le cas torique}

Nous montrons dans ce paragraphe que si $X$ est une variété de Fano torique de dimension $n$, on a $\rho_{X}\left(\iota_{X}-1\right) \leq n$ lorsque $\iota_{X} \geq \frac{1}{3} n+1$ ( $n$ arbitraire) ou $n \leq 7$.

4.1. Préliminaires. Soit $X$ une variété torique projective et lisse d'éventail $\Sigma_{X}$. Nous renvoyons à $[\mathrm{Fu} 93]$ ou [Od88] pour les fondements de la géométrie torique. Soit $G\left(\Sigma_{X}\right)$ l'ensemble des générateurs primitifs des cônes de dimension 1 dans $\Sigma_{X}$; son cardinal est $\rho_{X}+\operatorname{dim}(X)$. Notons $V(\sigma)$ l'adhérence de l'orbite correspondant à un élément $\sigma$ de $\Sigma_{X}$.

Rappellons ([Ba91], § 2 et [Ba99], § 2.1) qu'une collection primitive $P$ est un sous-ensemble de $G\left(\Sigma_{X}\right)$ minimal qui n'engendre pas un cône de $\Sigma_{X}$. À toute collection primitive $P=\left\{x_{1}, \ldots, x_{h}\right\}$ est associée sa relation primitive

$$
x_{1}+\cdots+x_{h}=a_{1} y_{1}+\cdots+a_{k} y_{k},
$$

où $\left\langle y_{1}, \ldots, y_{k}\right\rangle$ est le plus petit cône dans $\Sigma_{X}$ contenant le point $x_{1}+\cdots+x_{h}$ et 
où les $a_{i}$ sont des entiers strictement positifs. Le degré de $P$ est, par définition, $\operatorname{deg}(P)=h-\sum_{i} a_{i}$, et son ordre est $|P|=h$.

Le groupe $\mathrm{N}_{1}(X)$ des 1-cycles sur $X$ modulo équivalence numérique s'identifie au groupe des relations entre les éléments de $G\left(\Sigma_{X}\right)$; toute relation $\sum_{x \in G\left(\Sigma_{X}\right)} a_{x} x=0$ s'identifie à la classe d'équivalence numérique des 1-cycles dont l'intersection avec le diviseur $V(\langle x\rangle)$ est $a_{x}$.

En particulier, la relation primitive $x_{1}+\cdots+x_{h}-\left(a_{1} y_{1}+\cdots+a_{k} y_{k}\right)=0$ définit un élément $r(P)$ de $\mathrm{N}_{1}(X)$. Cette classe est toujours la classe d'un cycle effectif et $\operatorname{deg}(P)=-K_{X} \cdot r(P)$ ([Ba91], Theorem 2.15, [Ca03], Lemma 1.4). La variété $X$ est une variété de Fano si et seulement si toute relation primitive est de degré strictement positif et le pseudo-indice $\iota_{X}$ vérifie alors $|P|=h \geq \operatorname{deg}(P) \geq \iota_{X}$.

Une collection primitive $P$, ou sa relation associée $r(P)$, sont dites contractibles s'il existe un morphisme équivariant $\varphi: X \rightarrow W$ vers une variété torique complète $W$ telle que les courbes irréductibles contractées par $\varphi$ soient exactement celles dont la classe est dans $\mathbb{Q}_{\geq 0} r(P)$ ([Ca03], Definition 2.3). Avec les notations précédentes, le lieu exceptionnel de $\varphi$ est $A=V\left(\left\langle y_{1}, \ldots, y_{k}\right\rangle\right)$ et $\varphi_{\mid A}: A \rightarrow \varphi(A)$ est une fibration équivariante lisse en $\mathbb{P}^{h-1}$. Si $\operatorname{deg}(P)<2 \iota_{X}$, la collection $P$ est contractible ([Ca03], Theorem 4.1).

4.2. Fibrés projectifs. Soit $X$ une variété torique projective et lisse. La contraction $\varphi: X \rightarrow Y$ d'une arête numériquement effective de $\mathrm{NE}(X)$ est un fibré en espaces projectifs, $Y$ est une variété torique et $\varphi$ est équivariant.

Le lemme 2.5 permet de traiter facilement le cas des variétés toriques $X$ pour lesquelles toutes les collections primitives de $\Sigma_{X}$ sont disjointes. Cette condition combinatoire est équivalente à l'existence d'une suite $X=X_{1} \rightarrow X_{2} \rightarrow \cdots \rightarrow X_{r}$ où $X_{r}$ est un espace projectif et $X_{i}$ est une fibration en espaces projectifs sur $X_{i+1}$ pour $i=1, \ldots, r-1$ ([Ba91], Corollary 4.4). Rappelons enfin que les variétés toriques de nombre de Picard 2 sont précisément les fibrés en espaces projectifs sur l'espace projectif ([Kl88], Theorem 1) et qu'elles satisfont donc aux hypothèses du

Corollaire 4.3. Soit $X$ une variété de Fano torique. Si toutes les collections primitives de $\Sigma_{X}$ sont disjointes, on a $\rho_{X}\left(\iota_{X}-1\right) \leq \operatorname{dim}(X)$; de plus, si $\rho_{X}\left(\iota_{X}-1\right)$ $=\operatorname{dim}(X)$, on $a \quad X\left(\mathbb{P}^{\iota_{X}-1}\right)^{\rho_{X}}$.

Démonstration. Il existe donc des fibrations

$$
X=X_{1} \rightarrow X_{2} \rightarrow \cdots \rightarrow X_{r}=\mathbb{P}^{s_{r}}
$$

où $X_{i}$ est une fibration en espaces projectifs sur $X_{i+1}$ de fibre $\mathbb{P}^{s_{i}}$ pour $i=$ $1, \ldots, r-1$. En appliquant le lemme 2.5 , on obtient, par récurrence sur $i$, l'inégalité $\rho_{X}\left(\iota_{X}-1\right) \leq \operatorname{dim}(X)$. Supposons $\rho_{X}\left(\iota_{X}-1\right)=\operatorname{dim}(X)$. Comme $\rho_{X}=r$, $\operatorname{dim}(X)=s_{1}+\cdots+s_{r}$ et $\iota_{X}-1 \leq \min \left\{s_{1}, \ldots, s_{r}\right\}$, on a

$$
s_{1}+\cdots+s_{r}=r\left(\iota_{X}-1\right) \leq r \min \left\{s_{1}, \ldots, s_{r}\right\} \leq s_{1}+\cdots+s_{r},
$$


et donc $s_{1}=\cdots=s_{r}=\iota_{X}-1$. On vérifie enfin que $X$ est un produit d'espaces projectifs par récurrence descendante sur l'entier $1 \leq i \leq r-1$.

4.4. Le cas $3 \iota_{X} \geq \operatorname{dim}(X)+3$. Soit $X$ une variété de Fano torique. La preuve du résultat principal repose sur les deux lemmes suivants.

Lemme 4.5. Soit $A \subset X$ une sous-variété irréductible invariante de codimension $k$. Si $k \leq \iota_{X}-2$, alors $A$ est une variété de Fano de nombre de Picard $\rho_{X}$ et de pseudo-indice $\geq \iota_{X}-k$.

Démonstration. Il suffit de prouver le lemme pour $k=1$ et $\iota_{X} \geq 3$ : le résultat général s'ensuit par récurrence sur $k$. Soit donc $A=V(\langle x\rangle)$ un diviseur irréductible invariant et supposons $\iota_{X} \geq 3$. Si $P$ est une collection primitive dans $\Sigma_{A}$, ou bien $P$ est une collection primitive dans $\Sigma_{X}$ et $\operatorname{deg}_{A}(P) \geq \operatorname{deg}_{X}(P) \geq \iota_{X}$, ou bien $P \cup\{x\}$ est une collection primitive dans $\Sigma_{X}$, et $\operatorname{deg}_{A}(P)=\operatorname{deg}_{X}(P)-1 \geq \iota_{X}-1$. Comme $\iota_{X} \geq 2$, le diviseur $A$ est une variété de Fano de pseudo-indice $\geq \iota_{X}-1$. Puisque $\iota_{X} \geq 3$, il n'y a pas de collection primitive d'ordre $2(c f .4 .1): \Sigma_{A}$ a donc exactement un cône de dimension 1 de moins que $\Sigma_{X}$ et $\rho_{A}=\rho_{X}$.

Lemme 4.6. Soit $X$ une variété de Fano torique de dimension n, de pseudoindice $\iota_{X}$ et de nombre de Picard $\rho_{X}$. Si $4 \iota_{X}>n+4$, il existe dans $X$ une collection primitive contractible telle que la codimension du lieu exceptionnel de la contraction associée soit $\leq \iota_{X}-2$.

Démonstration. Supposons que toute relation primitive contractible de $X$ soit de la forme $x_{1}+\cdots+x_{h}=a_{1} y_{1}+\cdots+a_{k} y_{k}$ avec $k \geq \iota_{X}-1$. On a alors $h-k \geq h-\sum_{i} a_{i} \geq$ $\iota_{X}$, d'où $h \geq \iota_{X}+k \geq 2 \iota_{X}-1$. D'autre part, si $P$ est une collection primitive non contractible, on a $|P| \geq \operatorname{deg}(P) \geq 2 \iota_{X}$ (cf. 4.1). Il n'existe donc pas de collection primitive d'ordre $\leq 2 \iota_{X}-2$ dans $\Sigma_{X}$, de sorte que $f_{j-1}=\left(\begin{array}{c}f_{0} \\ j\end{array}\right)$ pour tout $j \leq 2 \iota_{X}-2$ (cf. 4.8). Puisque $X$ n'est pas isomorphe à $\mathbb{P}^{n}$, on a $2 \iota_{X}-2<[n / 2]+1$ par la proposition 4.11 , ce qui contredit les hypothèses.

Théorème 4.7. Soit $X$ une variété de Fano torique de dimension n, de pseudoindice $\iota_{X}$ et de nombre de Picard $\rho_{X}$. Si $3 \iota_{X} \geq n+3$, on a $\rho_{X}\left(\iota_{X}-1\right) \leq n$ (et donc $\left.\rho_{X} \leq 3\right)$; de plus, si $\rho_{X}\left(\iota_{X}-1\right)=n$, on a $X \simeq\left(\mathbb{P}^{\iota_{X}-1}\right)^{\rho_{X}}$.

Démonstration. Le théorème se montre par récurrence sur $\rho_{X}$. Si $\rho_{X}=1$, on a $X \simeq \mathbb{P}^{n}$ et le résultat est démontré. Sinon, d'après le lemme 4.6, il existe dans $X$ une relation primitive contractible qui s'écrit

$$
x_{1}+\cdots+x_{h}=a_{1} y_{1}+\cdots+a_{k} y_{k}
$$

avec $k \leq \iota_{X}-2$. Soit $A=V\left(\left\langle y_{1}, \ldots, y_{k}\right\rangle\right)$ le lieu exceptionnel de la contraction associée. D'après le lemme $4.5, A$ est une variété de Fano, $\rho_{A}=\rho_{X}$ et $\iota_{A} \geq \iota_{X}-k$. 
De plus, la contraction fait de $A$ une fibration $A \rightarrow B$ en $\mathbb{P}^{h-1}$, où $B$ est une variété de Fano torique satisfaisant $\rho_{B}=\rho_{X}-1, \operatorname{dim}(B)=n-k-h+1$ et $\iota_{B} \geq \iota_{A} \geq \iota_{X}-k$.

Vérifions l'inégalité $\iota_{B}>\frac{1}{3} \operatorname{dim}(B)+1$. Notons que $h \geq k+\iota_{X} \geq 2 k+2$ et que $\operatorname{dim}(B)=n-k-h+1 \leq n-3 k-1<n-3 k$, d'où

$$
\iota_{B} \geq \iota_{X}-k \geq \frac{1}{3} n+1-k=\frac{1}{3}(n-3 k)+1>\frac{1}{3} \operatorname{dim}(B)+1 .
$$

L'hypothèse de récurrence donne donc $\rho_{B}\left(\iota_{B}-1\right) \leq \operatorname{dim}(B)$ et $\rho_{B}=\rho_{X}-1 \leq 2$ puisque $\iota_{B}>\frac{1}{3} \operatorname{dim}(B)+1$. Finalement :

$$
\left(\rho_{X}-1\right)\left(\iota_{X}-k-1\right) \leq \rho_{B}\left(\iota_{B}-1\right) \leq \operatorname{dim}(B)=n-k-h+1
$$

d'où

$$
\rho_{X}\left(\iota_{X}-1\right)-n \leq \rho_{X} k+\iota_{X}-h-2 k \leq k+\iota_{X}-h \leq 0 .
$$

Supposons maintenant $\rho_{X}\left(\iota_{X}-1\right)=n$ et notons qu'il suffit de montrer $A=X$, c'est-à-dire $k=0$. En effet, $X$ est alors une fibration en espaces projectifs sur une variété de Fano torique $B$ satisfaisant $\rho_{B} \leq 2$ et le corollaire 4.3 s'applique. Supposons donc $k>0$. Les inégalités précédentes sont des égalités, d'où

$\iota_{B}=\iota_{A}=\iota_{X}-k, \rho_{B}\left(\iota_{B}-1\right)=\operatorname{dim}(B), \quad \rho_{X}=\rho_{A}=3, \quad \rho_{B}=2$ et $h=k+\iota_{X}$.

La variété $B$ est donc isomorphe à $\mathbb{P}^{\iota_{X}-k-1} \times \mathbb{P}^{\iota_{X}-k-1}$ (corollaire 4.3) et, puisque $A \rightarrow B$ est une fibration en $\mathbb{P}^{\iota_{X}+k+1}$ et $\iota_{A}=\iota_{X}-k$, la variété $A$ est isomorphe à $\mathbb{P}^{\iota_{X}+k-1} \times \mathbb{P}^{\iota_{X}-k-1} \times \mathbb{P}^{\iota_{X}-k-1}$. L'éventail $\Sigma_{A}$ est donc déterminé par les relations primitives

$$
\bar{x}_{1}+\cdots+\bar{x}_{\iota_{X}+k}=0, \bar{v}_{1}+\cdots+\bar{v}_{\iota_{X}-k}=0 \text { et } \bar{w}_{1}+\cdots+\bar{w}_{\iota_{X}-k}=0,
$$

où l'on a noté $\bar{u}$ le générateur de $\Sigma_{A}$ induit par l'élément $u \in G\left(\Sigma_{X}\right)$ tel que $\left\langle u, y_{1}, \ldots, y_{k}\right\rangle \in \Sigma_{X}$. On a nécessairement

$$
G\left(\Sigma_{X}\right)=\left\{x_{1}, \ldots, x_{\iota_{X}+k}, y_{1}, \ldots, y_{k}, v_{1}, \ldots, v_{\iota_{X}-k}, w_{1}, \ldots, w_{\iota_{X}-k}\right\}
$$

puisque $\rho_{X}=3$. Puisque toute classe extrémale de $X$ se restreint à une classe extrémale dans $A$, il doit y avoir dans $X$ trois relations primitives extrémales, de degré au moins $\iota_{X}$, dont les restrictions à $A$ sont les relations primitives ci-dessus. La seule possibilité est que les relations

$$
v_{1}+\cdots+v_{\iota_{X}-k}+y_{1}+\cdots+y_{k}=0 \text { et } w_{1}+\cdots+w_{\iota_{X}-k}+y_{1}+\cdots+y_{k}=0
$$

soient extrémales dans $X$, ce qui est absurde si $k>0$ car ces relations ne sont pas disjointes ([Ca03], Proposition 3.4).

4.8. Les petites dimensions. Dans ce paragraphe, on étudie les cas $\iota_{X}=[n / 2]$ et $\iota_{X}=[n / 2]-1$ lorsqu'ils ne sont pas couverts par le théorème 4.7 , c'est-à-dire 
respectivement pour $n \leq 7$ et $n \leq 13$. Nous en déduirons que l'inégalité $(*)$ est toujours verifiée si $n \leq 7$. Nous supposerons dans la suite $n \geq 4$.

Proposition 4.9. Soit $X$ une variété de Fano torique de dimension $n \leq 13$ et de pseudo-indice $[n / 2]$ ou $[n / 2]-1$. Si $X$ n'est pas une fibration en $\mathbb{P}^{\iota_{X}-1}$, l'entier $\rho_{X}$ satisfait les inégalités suivantes :

\begin{tabular}{|c|c|c|c|c|c|c|c|c|c|c|}
\cline { 2 - 7 } \multicolumn{1}{c|}{} & $n=4$ & $n=5$ & $n=6$ & $n=7$ & $n=8$ & $n=9$ & $n=10$ & $n=11$ & $n=12$ & $n=13$ \\
\hline$\iota_{X}=\left[\frac{n}{2}\right]$ & $\rho_{X} \leq 2$ & $\rho_{X} \leq 2$ & $\rho_{X} \leq 2$ & $\rho_{X} \leq 2$ & \multicolumn{9}{l|}{} \\
\hline$\iota_{X}=\left[\frac{n}{2}\right]-1$ & \multicolumn{1}{|c}{} & $\rho_{X} \leq 4$ & $\rho_{X} \leq 4$ & $\rho_{X} \leq 3$ & $\rho_{X} \leq 3$ & $\rho_{X} \leq 2$ & $\rho_{X} \leq 3$ & $\rho_{X} \leq 2$ & $\rho_{X} \leq 2$ \\
\hline
\end{tabular}

Corollaire 4.10. Soit $X$ une variété de Fano torique de dimension $n \leq 13$ et de pseudo-indice $[n / 2]$ ou $[n / 2]-1$. Alors $\rho_{X}\left(\iota_{X}-1\right) \leq \operatorname{dim}(X)$ et on a égalité si et seulement si $X \simeq\left(\mathbb{P}^{\iota_{X}-1}\right)^{\rho_{X}}$.

Démonstration. L'inégalité $\rho_{X}\left(\iota_{X}-1\right) \leq \operatorname{dim}(X)$, dans les cas non couverts par le théorème 4.7 , est équivalente aux bornes du tableau ci-dessous :

\begin{tabular}{|c|c|c|c|c|c|c|c|c|c|c|}
\hline & $n=4$ & $n=5$ & $n=6$ & $n=7$ & $n=8$ & $n=9$ & $n=10$ & $n=11$ & $n=12$ & $n=13$ \\
\hline$\iota_{X}=\left[\frac{n}{2}\right]$ & $\rho_{X} \leq 4$ & $\rho_{X} \leq 5$ & $\rho_{X} \leq 3$ & $\rho_{X} \leq 3$ & & & & & & \\
\hline$\iota_{X}=\left[\frac{n}{2}\right]-1$ & & & $\rho_{X} \leq 6$ & $\rho_{X} \leq 7$ & $\rho_{X} \leq 4$ & $\rho_{X} \leq 4$ & $\rho_{X} \leq 3$ & $\rho_{X} \leq 3$ & $\rho_{X} \leq 3$ & $\rho_{X} \leq 3$ \\
\hline
\end{tabular}

Si $X$ n'est pas une fibration en $\mathbb{P}^{\iota_{X}-1}$, la proposition 4.9 implique $\rho_{X}\left(\iota_{X}-1\right) \leq$ $\operatorname{dim}(X)$ et il n'y a jamais égalité.

Si $X$ est une fibration en $\mathbb{P}^{\iota_{X}-1}$ sur $Z$, on $\operatorname{arim}(Z)=n+1-\iota_{X} \leq(n-1) / 2$ et $\iota_{Z} \geq \iota_{X} \geq \operatorname{dim}(Z)-1$. Si $\operatorname{dim}(Z) \geq 5$, la variété $Z$ est un espace projectif (cf. 4.12) et donc $\rho_{X}=2$ : le résultat est une conséquence du corollaire 4.3. Si $\operatorname{dim}(Z) \leq 4$, on a $\iota_{Z} \geq 3$ et $\rho_{Z} \leq 2$, de sorte que le corollaire 4.3 s'applique encore à $X$.

Soit $P_{X}$ le polytope associé à $X$, c'est-à-dire le polytope simplicial convexe engendré par les éléments de $G\left(\Sigma_{X}\right)$. Les faces de $P_{X}$ correspondent aux cônes de $\Sigma_{X}$. Soit $f_{j}$ le nombre de faces de dimension $j$ de $P_{X}$. Rappelons l'égalité $f_{0}=\left|G\left(\Sigma_{X}\right)\right|=\rho_{X}+n$. L'éventail $\Sigma_{X}$ n'a pas de collection primitive d'ordre $j<\iota_{X}$, autrement dit,

$$
f_{j-1}=\left(\begin{array}{c}
f_{0} \\
j
\end{array}\right) \quad \text { pour tout } j<\iota_{X}
$$

De plus, si $\Sigma_{X}$ possède une collection primitive $P=\left\{x_{1}, \ldots, x_{\iota_{X}}\right\}$ d'ordre $\iota_{X}$, la relation associée est nécessairement $x_{1}+\cdots+x_{\iota_{X}}=0$, puisque son degré est 
au moins $\iota_{X}$. Elle est contractible et la contraction associée est une fibration en $\mathbb{P}^{\iota_{X}-1}$. Finalement :

$$
f_{\iota_{X}-1}<\left(\begin{array}{c}
f_{0} \\
\iota_{X}
\end{array}\right) \text { si et seulement si } X \text { est une fibration en } \mathbb{P}^{\iota_{X}-1} \text {. }
$$

Nous renvoyons à [MMS71] pour les propriétés fondamentales des polytopes simpliciaux. Rappelons en particulier qu'il existe des relations linéaires entre les $f_{j}$, appelées relations de Dehn-Sommerville, de sorte que les nombres $f_{0}, \ldots, f_{[n / 2]-1}$ déterminent tous les $f_{j}$. Rappelons aussi le résultat suivant ([MMS71], Chap. 2, Proposition 24)

Proposition 4.11. Un polytope de dimension $n$ est un simplexe si et seulement si $f_{j-1}=\left(\begin{array}{c}f_{0} \\ j\end{array}\right)$ pour tout $j \leq[n / 2]+1$.

4.12. Remarquons en particulier que si $\iota_{X}>[n / 2]+1$, la relation (3) et la proposition entraînent que $X$ est isomorphe à $\mathbb{P}^{n}$. Cela redémontre dans le cas torique le théorème de Wiśniewski cité dans l'introduction.

Les deux lemmes suivants donnent des relations supplémentaires entre les nombres $f_{j}$.

Lemme 4.13. Soit $X$ une variété de Fano torique. Si $\iota_{X}>1$, toutes les collections primitives de $\Sigma_{X}$ d'ordre $\iota_{X}+1$ sont deux à deux disjointes.

Démonstration. Soit $P=\left\{x_{1}, \ldots, x_{\iota_{X}+1}\right\}$ une collection primitive d'ordre $\iota_{X}+1$. Elle est nécessairement contractible puisque $\operatorname{deg}(P) \leq \iota_{X}+1<2 \iota_{X}$ (cf. 4.1). Ou bien $\operatorname{deg}(P)=\iota_{X}+1$, la relation primitive $r(P)$ est $x_{1}+\cdots+x_{\iota_{X}+1}=0$ et $P$ est disjointe de toutes les autres collections primitives de $\Sigma_{X}([\mathrm{Ca} 03]$, Proposition 3.4), ou bien $\operatorname{deg}(P)=\iota_{X}$ et la relation primitive $r(P)$ est de la forme $x_{1}+\cdots+x_{\iota_{X}+1}=$ $y$. Supposons par l'absurde qu'il existe deux relations primitives

$$
x_{1}+\cdots+x_{r}+y_{1}+\cdots+y_{s}=z \quad \text { et } \quad x_{1}+\cdots+x_{r}+u_{1}+\cdots+u_{s}=v
$$

avec $r+s=\iota_{X}+1$ et $r>0$. Comme $P$ est contractible, $\left\{y_{1}, \ldots, y_{s}, v\right\}$ doit contenir une collection primitive ([Ca03], Proposition 3.4), de sorte que $s+1 \geq \iota_{X}$ et $r \leq 2$. Si $r=2$ et $s=\iota_{X}-1$, la collection $\left\{y_{1}, \ldots, y_{s}, v\right\}$ est primitive, de relation associée $y_{1}+\cdots+y_{s}+v=0$, ce qui est absurde. Si $r=1$ et $s=\iota_{X}$, la collection $\left\{y_{1}, \ldots, y_{s}, v\right\}$ est primitive, de relation associée $y_{1}+\cdots+y_{s}+v=w$. Par ce qui précède, on a $s=1$ et $\iota_{X}=1$, ce qui est exclu par l'hypothèse.

Lemme 4.14. Si $X$ est une variété de Fano torique de dimension $n$, on a

$$
12 f_{n-3} \geq\left(3 n+\iota_{X}-5\right) f_{n-2} .
$$

Démonstration. Posons $d_{X}=\sum \operatorname{deg}\left(N_{C / X}\right)$, où la somme porte sur toutes les 
courbes invariantes de $X$. Si $C$ est une courbe invariante, on a $\operatorname{deg}\left(N_{C / X}\right)=$ $-K_{X} \cdot C-2 \geq \iota_{X}-2$, de sorte que $d_{X} \geq\left(\iota_{X}-2\right) f_{n-2}$. D'autre part, on a $d_{X}=12 f_{n-3}-3(n-1) f_{n-2}$ ([Ba99], Theorem 2.3.7), d'où l'inégalité cherchée.

Démonstration de la proposition 4.9. Posons $k=[n / 2]$ et supposons $\iota_{X}=k$. Comme $X$ n'est pas une fibration en $\mathbb{P}^{\iota_{X}-1}$, on a $f_{j-1}=\left(\begin{array}{c}f_{0} \\ j\end{array}\right)$ pout tout $j \leq k$. Les relations de Dehn-Sommerville permettent d'exprimer $f_{k}$ à l'aide de $f_{0}$ ([MMS71], $\oint 2.4)$ :

$$
\begin{aligned}
& f_{k}=\sum_{j=0}^{k-1}(-1)^{k-j-1} \frac{j+1}{k+1}\left(\begin{array}{c}
2 k-j \\
k
\end{array}\right)\left(\begin{array}{c}
f_{0} \\
j+1
\end{array}\right) \quad \text { si } n \text { est pair, } \\
& f_{k}=\sum_{j=-1}^{k-1}(-1)^{k-j-1}\left(\begin{array}{c}
2 k-j+1 \\
k+1
\end{array}\right)\left(\begin{array}{c}
f_{0} \\
j+1
\end{array}\right) \quad \text { si } n \text { est impair. }
\end{aligned}
$$

Le lemme 4.13 donne l'inégalité

$$
\left(\begin{array}{c}
f_{0} \\
k+1
\end{array}\right)-f_{k} \leq \frac{f_{0}}{k+1}
$$

d'où les bornes de la première ligne du tableau.

Supposons $\iota_{X}=k-1$ et que $X$ n'est pas une fibration en $\mathbb{P}^{\iota_{X}-1}$. On a alors

$$
f_{j-1}=\left(\begin{array}{c}
f_{0} \\
j
\end{array}\right) \text { pour tout } j \leq k-1 \quad \text { et } \quad\left(\begin{array}{c}
f_{0} \\
k
\end{array}\right)-f_{k-1} \leq \frac{f_{0}}{k} .
$$

Les relations de Dehn-Sommerville permettent d'exprimer tous les $f_{j}$ avec $j \geq k$ à l'aide de $f_{0}$ et $f_{k-1}$. Si $n$ est pair, on a ([MMS71], $\S 2.4$ )

$$
\begin{gathered}
f_{n-2}=k f_{k-1}+\sum_{j=1}^{k-1}(-1)^{j} \frac{k-j}{k+j-1}\left((k-1)\left(\begin{array}{c}
k+j \\
k
\end{array}\right)+\left(\begin{array}{c}
k+j-1 \\
k
\end{array}\right)\right)\left(\begin{array}{c}
f_{0} \\
k-j
\end{array}\right) \text { et } \\
f_{n-3}=\left(\begin{array}{c}
k \\
2
\end{array}\right) f_{k-1}+\sum_{j=1}^{k-1}(-1)^{j} \frac{k-j}{k+j-2}\left(\left(\begin{array}{l}
k \\
2
\end{array}\right)\left(\begin{array}{c}
k+j \\
k
\end{array}\right)\right. \\
\left.+(k-2)\left(\begin{array}{c}
k+j-1 \\
k
\end{array}\right)+\left(\begin{array}{c}
k+j-2 \\
k
\end{array}\right)\right)\left(\begin{array}{c}
f_{0} \\
k-j
\end{array}\right) .
\end{gathered}
$$

Et si $n$ est impair, on a

$$
\begin{gathered}
f_{n-2}=(2 k+1) f_{k-1}+\sum_{j=1}^{k}(-1)^{j} \frac{2 k+1}{k+j}\left(k\left(\begin{array}{c}
k+j+1 \\
k+1
\end{array}\right)+\left(\begin{array}{c}
k+j \\
k+1
\end{array}\right)\right)\left(\begin{array}{c}
f_{0} \\
k-j
\end{array}\right) \text { et } \\
f_{n-3}=k^{2} f_{k-1}+\sum_{j=1}^{k}(-1)^{j} \frac{2 k}{k+j-1}\left(\left(\begin{array}{l}
k \\
2
\end{array}\right)\left(\begin{array}{c}
k+j+1 \\
k+1
\end{array}\right)\right. \\
\left.+(k-1)\left(\begin{array}{c}
k+j \\
k+1
\end{array}\right)+\left(\begin{array}{c}
k+j-1 \\
k+1
\end{array}\right)\right)\left(\begin{array}{c}
f_{0} \\
k-j
\end{array}\right) .
\end{gathered}
$$


Finalement, en appliquant le lemme 4.14, on obtient $f_{k-1} \leq \Psi_{k}\left(f_{0}\right)$, où, si $n$ est pair,

$$
\begin{aligned}
\Psi_{k}\left(f_{0}\right) & =\frac{k^{3}-k^{2}+12}{k^{2}}\left(\begin{array}{c}
f_{0} \\
k-1
\end{array}\right) \\
& +\sum_{j=2}^{k-1} \frac{(-1)^{j-1}(k-j)(k+j-3) !}{k(k !)(j !)}((k+j-1)(k+j-2) k+12 j)\left(\begin{array}{c}
f_{0} \\
k-j
\end{array}\right)
\end{aligned}
$$

et, si $n$ est impair,

$$
\begin{aligned}
& \Psi_{k}\left(f_{0}\right)=\frac{2 k^{3}+3 k^{2}-2 k+21}{(k-1)(2 k+3)}\left(\begin{array}{c}
f_{0} \\
k-1
\end{array}\right) \\
& \quad+\sum_{j=2}^{k} \frac{(-1)^{j-1}(k+j-2) !}{(k-1)(2 k+3)(k !)(j !)}\left(2 k^{4}+(4 j-1) k^{3}\right. \\
& \left.\quad+2\left(j^{2}-2\right) k^{2}+\left(j^{2}+17 j+3\right) k+3 j(1-j)\right)\left(\begin{array}{c}
f_{0} \\
k-j
\end{array}\right) .
\end{aligned}
$$

On déduit alors du lemme 4.13 l'inégalité

$$
\left(\begin{array}{l}
f_{0} \\
k
\end{array}\right)-\frac{f_{0}}{k}-\Psi_{k}\left(f_{0}\right) \leq 0
$$

ce qui donne les bornes de la deuxième ligne du tableau par une étude directe.

\section{Chaînes de courbes rationnelles}

Soit $X$ une variété projective, lisse et connexe et soient $V^{1}, \ldots, V^{k}$ des familles propres irréductibles de courbes rationnelles irréductibles sur $X$ (cf. 3.14). Fixons $x \in \operatorname{lieu}\left(V^{1}\right)$, posons $\operatorname{lieu}\left(V^{1}\right)_{x}=\operatorname{lieu}\left(V_{x}^{1}\right)$ et $Z_{1}=\mathcal{U}_{x}^{1}(c f .3 .14)$ et, avec les notations

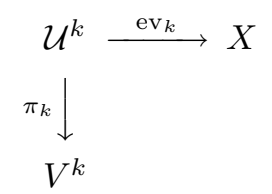

définissons par récurrence pour $k \geq 2$ :

$$
\begin{aligned}
Z_{k} & =\pi_{k}^{-1}\left(\pi_{k}\left(\operatorname{ev}_{k}^{-1}\left(\operatorname{lieu}\left(V^{1}, \ldots, V^{k-1}\right)_{x} \cap \operatorname{lieu}\left(V^{k}\right)\right)\right)\right) \\
\operatorname{lieu}\left(V^{1}, \ldots, V^{k}\right)_{x} & =\operatorname{ev}_{k}\left(Z_{k}\right) \\
U_{x}^{k} & =\operatorname{lieu}\left(V^{1}, \ldots, V^{k}\right)_{x}-\operatorname{lieu}\left(V^{1}, \ldots, V^{k-1}\right)_{x}
\end{aligned}
$$

Le fermé lieu $\left(V^{1}, \ldots, V^{k}\right)_{x}$ est donc l'ensemble des points $y$ de $X$ tels qu'il existe des courbes $C^{1}, \ldots, C^{k}$ avec

- $\left[C^{j}\right] \in V^{j}$; 
- les intersections $C^{1} \cap C^{2}, \ldots, C^{k-1} \cap C^{k}$ ne sont pas vides;

- $x \in C^{1}$ et $y \in C^{k}$.

Lemme 5.1. Si les classes des familles $V^{1}, \ldots, V^{k}$ dans $\mathrm{N}_{1}(X)_{\mathbb{Q}}$ sont linéairement indépendantes et que lieu $\left(V^{1}, \ldots, V^{k}\right)_{x} n^{\prime}$ 'est pas vide,

(a) le morphisme d'évaluation $\mathrm{ev}_{k}: Z_{k} \rightarrow \operatorname{lieu}\left(V^{1}, \ldots, V^{k}\right)_{x}$ est fini au-dessus de l'ouvert $U_{x}^{k}$, qui n'est pas vide;

(b) toute courbe tracée sur lieu $\left(V^{1}, \ldots, V^{k}\right)_{x}$ est algébriquement équivalente dans cette variété à une combinaison linéaire à coefficients rationnels de courbes dans $V^{1}, \ldots, V^{k}$.

Démonstration. Le résultat se montre par récurrence sur l'entier $k \geq 1$. Si $k=1$, le point (a) est une conséquence du lemme de cassage de Mori ([Mo79], Theorem 6) et le point (b) est le lemme 3.15. Supposons $k \geq 2$.

Démontrons (a). Soit $z$ un point de lieu $\left(V^{1}, \ldots, V^{k-1}\right)_{x} \cap \operatorname{lieu}\left(V^{k}\right)$, qui n'est pas vide par hypothèse. Si lieu $\left(V_{z}^{k}\right) \subset \operatorname{lieu}\left(V^{1}, \ldots, V^{k-1}\right)_{x}$, les classes des familles $V^{1}, \ldots, V^{k}$ sont linéairement dépendantes dans $\mathrm{N}_{1}(X)_{\mathbb{Q}}$ par hypothèse de récurrence, ce qui est absurde. Ainsi, $U_{x}^{k}$ n'est pas vide. Si un point $y$ de $U_{x}^{k}$ vérifie $\operatorname{dim}\left(\mathrm{ev}_{k}^{-1}(y) \cap Z_{k}\right) \geq 1$,

- soit $\operatorname{dim}\left(\operatorname{lieu}\left(V_{y}^{k}\right) \cap \operatorname{lieu}\left(V^{1}, \ldots, V^{k-1}\right)_{x}\right) \geq 1$ et, à nouveau par hypothèse de récurrence, les classes des familles $V^{1}, \ldots, V^{k}$ sont linéairement dépendantes dans $\mathrm{N}_{1}(X)_{\mathbb{Q}}$, ce qui contredit l'hypothèse;

- soit il existe une famille de dimension 1 de courbes rationnelles de $V^{k}$ passant toutes par deux points distincts de $X$ fixés, ce qui, par le lemme de cassage ([Mo79], Theorem 6), est absurde puisque la famille $V^{k}$ est propre.

Démontrons (b). Soit $C$ une courbe tracée sur lieu $\left(V^{1}, \ldots, V^{k}\right)_{x}$ telle que $[C] \notin V^{k}$. Si $C$ est dans lieu $\left(V^{1}, \ldots, V^{k-1}\right)_{x}$, l'hypothèse de récurrence permet de conclure. Supposons donc que $C$ n'est pas contenue dans $\operatorname{lieu}\left(V^{1}, \ldots, V^{k-1}\right)_{x}$. Soit $\mathcal{C} \subset \mathrm{ev}_{k}^{-1}(C) \cap Z_{k}$ une courbe irréductible dominant $C$, soit $\mathcal{S} \subset \mathcal{U}^{k}$ la surface irréductible $\pi_{k}^{-1}\left(\pi_{k}(\mathcal{C})\right)$, soit $S$ la surface $\operatorname{ev}_{k}(\mathcal{S}) \subset X$ et soit $\mathcal{C}^{\prime} \subset \mathcal{S}$ une courbe dominant $S \cap$ lieu $\left(V^{1}, \ldots, V^{k-1}\right)_{x}$. Toute courbe tracée sur $\mathcal{S}$ est algébriquement équivalente dans $\mathcal{S}$ à une combinaison linéaire à coefficients rationnels de la multisection $\mathcal{C}^{\prime}$ et d'une fibre de $\pi_{k \mid \mathcal{S}}: \mathcal{S} \rightarrow \pi_{k}(\mathcal{S})$ ([Ko96], II.4.19). Toute courbe tracée sur $S$ est donc algébriquement équivalente dans $S$, donc dans $\operatorname{lieu}\left(V^{1}, \ldots, V^{k}\right)_{x}$, à une combinaison linéaire à coefficients rationnels $\operatorname{de~} \mathrm{ev}_{k}\left(\mathcal{C}^{\prime}\right)$ et d'une courbe de $V^{k}$ ([Ko96], II.4.4.2). Il reste à remarquer que $\operatorname{ev}_{k}\left(\mathcal{C}^{\prime}\right) \subset \operatorname{lieu}\left(V^{1}, \ldots, V^{k-1}\right)_{x}$ : l'hypothèse de récurrence permet de conclure.

On en déduit le résultat suivant.

Théorème 5.2. Si les classes des familles $V^{1}, \ldots, V^{k}$ dans $\mathrm{N}_{1}(X)_{\mathbb{Q}}$ sont linéairement indépendantes, lieu $\left(V^{1}, \ldots, V^{k}\right)_{x}$ est vide ou de dimension $\geq-\sum_{j=1}^{k} K_{X} \cdot V^{j}-k$. 
Démonstration. On procède par récurrence sur l'entier $k \geq 1$. Si $k=1$, l'estimation sur la dimension a déja été mentionnée en 3.15. Supposons $k \geq 2$ et que lieu $\left(V^{1}, \ldots, V^{k}\right)_{x}$ n'est pas vide. Le lemme précédent donne

$$
\operatorname{dim}\left(\operatorname{lieu}\left(V^{1}, \ldots, V^{k}\right)_{x}\right) \geq \operatorname{dim}\left(Z_{k}\right) .
$$

Si $y$ est un point général de $\operatorname{lieu}\left(V^{1}, \ldots, V^{k-1}\right)_{x} \cap \operatorname{lieu}\left(V^{k}\right)$, on a $\operatorname{dim}\left(\operatorname{ev}_{k}^{-1}(y)\right)=$ $\operatorname{dim}\left(V_{y}^{k}\right)$ et, si l'on note

$$
W_{k}=\operatorname{ev}_{k}^{-1}\left(\operatorname{lieu}\left(V^{1}, \ldots, V^{k-1}\right)_{x} \cap \operatorname{lieu}\left(V^{k}\right)\right),
$$

on a, en utilisant l'hypothèse de récurrence,

$$
\begin{aligned}
\operatorname{dim}\left(W_{k}\right) & =\operatorname{dim}\left(V_{y}^{k}\right)+\operatorname{dim}\left(\operatorname{lieu}\left(V^{1}, \ldots, V^{k-1}\right)_{x} \cap \operatorname{lieu}\left(V^{k}\right)\right) \\
& \geq \operatorname{dim}\left(V_{y}^{k}\right)-\sum_{j=1}^{k-1} K_{X} \cdot V^{j}-(k-1)+\operatorname{dim}\left(\operatorname{lieu}\left(V^{k}\right)\right)-n \\
& \geq \operatorname{dim}\left(V_{k}\right)-\sum_{j=1}^{k-1} K_{X} \cdot V^{j}-k+2-n,
\end{aligned}
$$

d'où, par l'inégalité (1) de 3.14,

$$
\operatorname{dim}\left(W_{k}\right) \geq-\sum_{j=1}^{k} K_{X} \cdot V^{j}-k-1 .
$$

Comme $\operatorname{dim}\left(Z_{k}\right)=\operatorname{dim}\left(W_{k}\right)+1$, le lemme précédent permet de conclure.

On déduit du théorème le corollaire suivant.

Corollaire 5.3. Soit $X$ une variété de Fano de nombre de Picard $\rho_{X}$ et de pseudoindice $\iota_{X}$. S'il existe des familles propres irréductibles $V^{1}, \ldots, V^{\rho_{X}}$ de courbes rationnelles irréductibles sur $X$ dont les classes dans $\mathrm{N}_{1}(X)_{\mathbb{Q}}$ sont linéairement indépendantes et que lieu $\left(V^{1}, \ldots, V^{\rho_{X}}\right)_{x}$ n'est pas vide, on a $\rho_{X}\left(\iota_{X}-1\right) \leq \operatorname{dim}(X)$.

Il n'est évidemment pas facile d'assurer l'existence de familles propres de courbes rationnelles irréductibles sur $X$ vérifiant les conditions du corollaire précédent. $\mathrm{Si}$ $R \subset \mathrm{NE}(X)$ est une arête, les courbes rationnelles irréductibles dont la classe appartient à $R$ et de degré anticanonique minimal forment une famille propre. En considérant ces familles de courbes rationnelles, on montre le résultat suivant.

Corollaire 5.4. Soit $X$ une variété de Fano homogène. On a $\rho_{X}\left(\iota_{X}-1\right) \leq$ $\operatorname{dim}(X)$.

Les résultats récents de Brion [Bri02] montrent que l'inégalité $\rho_{X}\left(\iota_{X}-1\right) \leq$ $\operatorname{dim}(X)$ est aussi valable si $X$ est une variété de Fano admettant une action d'un groupe algébrique semi-simple avec une unique orbite fermée. 


\section{Références}

[An85] T. Ando, On extremal rays of the higher dimensional varieties, Invent. Math. 81 (1985), 347-357.

[ABW92] M. Andreatta, E. Ballico and J. A. Wiśniewski, Vector bundles and adjunction, Internat. J. Math. 3 (1992), 331-340.

[AW98] M. Andreatta and J. A. Wiśniewski, Contractions of smooth varieties. II. Computations and applications, Boll. Unione Mat. Ital. Sez. B Artic. Ric. Mat. (8) 1 (1998), 343-360.

[Ba91] V. V. Batyrev, On the classification of smooth projective toric varieties, Tohoku Mathematical Journal 43 (1991), 569-585.

[Ba99] V. V. Batyrev, On the classification of toric Fano 4-folds, Journal of Mathematical Sciences (New York) 94 (1999), 1021-1050.

[BCW01] L. Bonavero, F. Campana and J. A. Wiśniewski, Variétés projectives complexes dont l'éclatée en un point est de Fano, C.R. Acad. Sci. Paris, Ser. I 334 (2002), 463-468.

[Bri02] M. Brion, The cone of effective one-cycles of certain G-varieties, Prépublication math.AG/0211027 (2002).

[Ca03] C. Casagrande, Contractible classes in toric varieties, Math. Zeitschrift 243 (2003), 99-126.

[CMS00] K. Cho, Y. Miyaoka and N. Shepherd-Barron, Characterizations of projective space and applications to complex symplectic manifolds, Higher dimensional birational geometry (Kyoto, 1997), 1-88, Adv. Stud. Pure Math. 35, Math. Soc. Japan, Tokyo, 2002.

[Fu93] W. Fulton. Introduction to toric varieties, Annals of mathematics studies 131, Princeton University Press, 1993.

[GHS03] T. Graber, J. Harris and J. Starr, Families of rationally connected varieties, J. Amer. Math. Soc. 16 (2003), 57-67.

[Gr68] A. Grothendieck, Le groupe de Brauer. I. Algèbres d'Azumaya et interprétations diverses, Dix exposés sur la cohomologie des schémas, 46-66, North-Holland, Amsterdam; Masson, Paris, 1968.

[IP99] V. A. Iskovskikh and Yu. G. Prokhorov, Fano varieties, Algebraic geometry V, Encyclopaedia Math. Sci. 47, Springer-Verlag, Berlin, 1999.

[Ka89] Y. Kawamata, Small contractions of four dimensional algebraic manifolds, Math. Ann. 284 (1989), 595-600.

[Ke01] S. Kebekus, Characterizing the projective space after Cho, Miyaoka and ShepherdBarron, Festschrift in honor of Hans Grauert. 147-155, Complex geometry (Göttingen, 2000), Springer, Berlin, 2002.

[Kl88] P. Kleinschmidt, A classification of toric varieties with few generators, Aequationes Math. 35 (1988), 254-266.

[Ko96] J. Kollár, Rational curves on algebraic varieties, Ergebnisse der Mathematik und ihre Grenzgebiete, 3. Folge, 032, Springer-Verlag, 1996.

[KMM92] J. Kollár, Y. Miyaoka and S. Mori, Rational Connectedness and Boundedness of Fano Manifolds. J. Diff. Geom. 36 (1992), 765-769.

[La83] R. Lazarsfeld, Some applications of the theory of positive vector bundles, Complete intersections (Acireale, 1983), 29-61, Lecture Notes in Math. 1092, Springer-Verlag, Berlin, 1984. 
[MMS71] P. McMullen and G. C. Shephard, Convex Polytopes and the Upper Bound Conjecture, London Mathematical Society Lecture Note Series 3, Cambridge University Press, 1971.

[Mo79] S. Mori, Projective manifolds with ample tangent bundles, Ann. of Math. 110 (1979), $593-606$.

[Mo82] S. Mori, Threefolds whose canonical bundles are not numerically effective, Ann. of Math. 116 (1982), 133-176.

[MM81] S. Mori and S. Mukai, Classification of Fano 3-folds with $B_{2} \geq 2$, Manuscripta Math. 36 (1981), 147-162.

[Mu88] S. Mukai, Problems on characterization of the complex projective space, Birational geometry of algebraic varieties. Open problems, The 23rd Intern. Sympos. Division of Math., The Taniguchi Foundation. Kataka, 1988.

[Od88] T. Oda, Convex bodies and algebraic geometry, an introduction to the theory of toric varieties, Ergebnisse der Mathematik und ihrer Grenzgebiete, 3. Folge, 015, SpringerVerlag, 1988.

[Wi90] J. A. Wiśniewski, On a conjecture of Mukai, Manuscripta Math. 68 (1990), 135-141.

[Wi91] J. A. Wiśniewski, On contractions of extremal rays of Fano manifolds, J. reine angew. Math. 417 (1991), 141-157.

L. Bonavero et S. Druel Institut Fourier

UFR de Mathématiques

Université de Grenoble 1

UMR 5582, BP 74

38402 Saint Martin d'Hères

France

e-mail : bonavero@ujf-grenoble.fr

e-mail : druel@ujf-grenoble.fr

O. Debarre

Mathématique - IRMA - UMR 7501

Université Louis Pasteur

7, rue René Descartes

67084 Strasbourg

France

e-mail : debarre@math.u-strasbg.fr

(Received: May 6, 2002)
C. Casagrande

Università di Roma "La Sapienza"

Dipartimento di Matematica

Piazzale Aldo Moro, 2

00185 Roma

Italia

e-mail : ccasagra@mat.uniroma1.it 